\title{
Electromagnetically controlled multi-scale flows
}

\author{
By L. ROSSI ${ }^{1}$, J. C. VASSILICOS AND Y. HARDALUPAS \\ ${ }^{1}$ Department of Aeronautics, Imperial College London SW7 2AZ, UK \\ ${ }^{2}$ Department of Mechanical Engineering, Imperial College London SW7 2AZ, UK
}

(Received 28 July 2005 and in revised form 7 December 2005)

We generate a class of multi-scale quasi-steady laminar flows in the laboratory by controlling a quasi-two-dimensional shallow-layer brine flow by multi-scale Lorentz body forcing. The flows' multi-scale topology is invariant over a broad range of Reynolds numbers, $R e_{2 D}$ from 600 to 9900 . The key multi-scale aspects of this flow associated with its multi-scale hyperbolic stagnation-point structure are highlighted. Our multiscale flows are laboratory simulations of quasi-two-dimensional turbulent-like flows, and they have a power-law energy spectrum $E(k) \sim k^{-p}$ over a range $2 \pi / L<k<2 \pi / \eta$ where $p$ lies between the values $5 / 3$ and 3 which are obtained in a two-dimensional turbulence that is forced at the small scale $\eta$ or at the large scale $L$, respectively. In fact, in the present set-up, $p+D_{s}=3$ in agreement with a previously established formula; $D_{s} \approx 0.5$ is the fractal dimension of the set of stagnation points and $p \approx 2.5$. The two exponents $D_{s}$ and $p$ are controlled by the multi-scale electromagnetic forcing over the entire range of scales between $L$ and $\eta$ for a broad range of Reynolds numbers with separate control over $L / \eta$ and Reynolds number. The pair dispersion properties of our multi-scale laminar flows are also controlled by their multi-scale hyperbolic stagnation-point topology which generates a sequence of exponential separation processes starting from the smaller-scale hyperbolic points and ending with the larger ones. The average mean square separation $\overline{\Delta^{2}}$ has an approximate power law behaviour $\sim t^{\gamma}$ with 'Richardson exponent' $\gamma \approx 2.45$ in the range of time scales controlled by the hyperbolic stagnation-points. This exponent is itself controlled by the multi-scale quasi-steady hyperbolic stagnation-point topology of the flow.

\section{Introduction}

Turbulence and mixing are two closely related research areas. Turbulence is a natural mixer in many astrophysical, geophysical, environmental and industrial flows; and mixing statistics of turbulent flows bare the imprint of various turbulent velocity field properties. In this paper, we present a way to use electromagnetic (EM) flow control over many scales so as to simulate and study turbulent-like flows and turbulent-like mixing in the laboratory.

\subsection{Turbulent flows}

A central property of turbulent flows is that their energy spectra are continuous and in fact power-law functions of wavenumber over appropriate intermediate ranges of scales. In homogeneous isotropic turbulence, we know from Kolmogorov's seminal contributions of 1941 and the many experimental works which followed, that the energy spectrum's shape is not too far from $E(k) \sim k^{-5 / 3}$ in the inertial range (see Frisch 1995; Mathieu \& Scott 2000; Pope 2000; Davidson 2004). A particular vein of turbulence research starting with Novikov, Mandelbrot, Frisch and Parisi in the 
1970s and 1980s (see Frisch 1995) has sought to relate the power law shape of $E(k)$ to the multi-scale nature of actual flow-field realizations which can be directly measured and characterized in terms of fractal dimensions. For example, if the statistics of velocity field increments are assumed to be homogeneous, isotropic and Gaussian, the energy spectrum $E(k) \sim k^{-p}$ is related to the fractal (in fact Hausdorff) co-dimension $D$ of iso-surfaces of velocity components by $p+2 D=3$ (Orey 1970). Turbulent velocity increments being non-Gaussian in three-dimensional turbulence (see Frisch 1995), such a relation is not of obvious relevance to turbulence, but it does illustrate the type of relation sought which is between a scaling exponent such as $p$ characterizing the continuous power-law energy spectrum (or more generally, the scaling exponents characterizing power-law structure functions) and one or more fractal dimensions characterizing the multi-scale geometry of flow realizations (see Frisch 1995 for a discussion of fractal and multifractal models). These flow realizations do not need to be as disordered as assumed for $p+2 D=3$; Lundgren (1982) has shown that flow realizations consisting of strained spiral vortices randomly distributed in space and at different stages of their time-development can also produce a $k^{-5 / 3}$ spectral signature. Khan \& Vassilicos (2002) laboured this point in the spirit of the fractal approaches cited in Frisch (1995) and calculated the scaling exponents of scalar structure functions in a two-dimensional flow consisting of many independent vortices which are persistent in the sense that they stay still as they advect the scalar around them. They found these scaling exponents to be linear functions of the fractal dimension (in fact Kolmogorov capacity) of the spiral interfacial patterns generated by these vortices on the scalar field.

The spirit behind the bulk of these works is to interpret continuous power-law spectra and structure functions as resulting from a quantifiable multi-scale geometry of field realizations, whether disordered as in Gaussian fields or organized as in spirals. In fact, in the context of such theories, the dependence of scalar or kinetic energy dissipation rates on Péclet or Reynolds numbers can also be controlled by fractal dimensions of multi-scale fields (Frisch 1995; Vassilicos 2002).

What better way is there to test the fundamental principle underlying these approaches than to attempt to generate fully controlable multi-scale flows in the laboratory and measure their resulting energy spectra? This cannot be done with turbulent flows because we do not fully control nor fully understand their multi-scale geometry and topology. Ideally, we want to generate in the laboratory multi-scale flows characterized by one or more fractal dimensions or other scaling parameters which we can vary at will so as to monitor the effects of their changes on spectra, structure functions, dissipation rates, etc.

Laboratory experiments where a single strained spiral vortex is generated in a carefully controlled manner have already been performed and have shown that the energy spectrum of such individual vortices with internal multi-scale flow structure is continuous and power-law shaped (Cuypers, Maurel \& Petitjeans 2003). The authors of these experiments have even documented the qualitative relation between the scaling exponent $p$ of the spectrum and the spatio-temporal structure of the flow. However, it is not clear how one might be able to fully control and modify the exponent $p$ at will in these spiral vortex experiments.

Here we chose to generate multi-scale fractal flows rather than multi-scale spiral flows. The only authors known to us who have considered how a fractal streamline pattern might look like for an incompressible flow are Fung \& Vassilicos (1998) and Moffatt (2001). Three-dimensional flows being hard to visualize, they have done so only for two-dimensional incompressible flows and their qualitative schematic is 


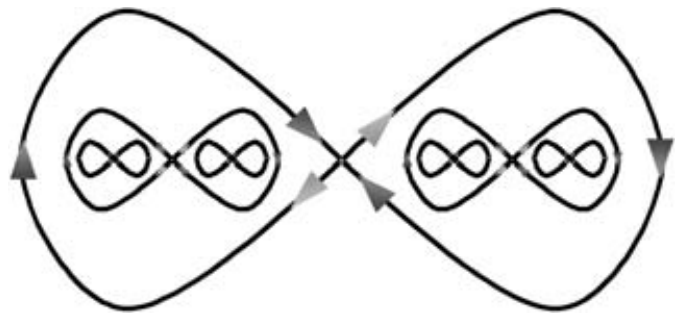

FIGURE 1. Schematic of a fractal (multi-scale) flow based on an 8 in 8 topology.

reproduced in figure 1. Davila \& Vassilicos (2003) pointed out that a straightforward way to quantify the multi-scale property of such a schematic streamline topology is to count how the number of stagnation points increases as the fractal range of scales increases. Specifically, Fung \& Vassilicos (1998) observed that realizations of velocity fields with statistically homogeneous, isotropic and Gaussian increments have a multiscale streamline topology which is qualitatively well described by figure 1 when they are two-dimensional; and Davila \& Vassilicos (2003) showed that the number density $n_{s}$ of the stagnation points (points where all velocity components vanish) scales as $(L / \eta)^{D_{s}}$ where $D=D_{s} / d$ ( $d$ being the dimension of the embedding space, i.e. $d=2$ or 3 ) and $L$ and $\eta$ are, respectively, the outer and inner length scales defining the intermediate range where $E(k) \sim k^{-p}$ holds. Thus, by using Orey's (1970) relation $p+2 D=3$, they were led to

$$
p+2 D_{s} / d=3 \text {. }
$$

Surprisingly, direct numerical simulations (DNS) of three-dimensional homogeneous isotropic turbulence yield $D_{s}=2$ (Davila \& Vassilicos 2003) and DNS of inverse cascading two-dimensional homogeneous isotropic turbulence yield $D_{s}=4 / 3$ (Goto \& Vassilicos 2004) in agreement with (1.1) and $p=5 / 3$ in both cases. In fact, the DNS of Goto \& Vassilicos (2004) even confirmed the 'cat's eyes within cat's eyes' multi-scale streamline structure of figure 1 . The statistics of turbulent velocity increments being non-Gaussian (Frisch 1995), the success of (1.1) in isotropic turbulence might be accounted for by the extreme sensitivity that stagnation points have on the slightest randomness in the multi-scale velocity field thus causing their number density to behave as if that field had Gaussian increments.

We therefore have a multi-scale fractal flow topology which in two dimensions looks schematically as in figure 1 and in two or three dimensions is characterized by the multi-scale nature of its stagnation points which is quantified by

$$
n_{s} \sim(L / \eta)^{D_{s}} .
$$

If we can find a way to generate such a flow in the laboratory, its energy spectrum should be continuous and power-law shaped with its scaling exponent $p$ determined by our choice of $D_{s}$ via equation (1.1). Such a multi-scale flow might not be without some likeness to isotropic homogeneous Navier-Stokes turbulence which also obeys (1.1).

\subsection{Mixing}

More importantly perhaps, a multi-scale flow based on the schematic given in figure 1 would incorporate some of the essential ingredients of two recent models of turbulent diffusion: the Kraichnan model of scalar turbulence (see review by Falkovich, Gawedzki \& Vergassola 2001) where the statistics of velocity field increments are 
assumed to be homogeneous, isotropic and Gaussian so that the energy spectrum is continuous and power-law shaped with $p$ given by (1.1); and the turbulent pairdiffusion model of Davila \& Vassilicos (2003), Goto \& Vassilicos (2004), Goto et al. (2005) and Osborne et al. (2006) where stagnation points in the frame where the mean flow is zero are assumed to be responsible for pair-separation statistics because they are shown to be statistically long-lived compared to all time scales of the turbulence and slowly moving compared to fluid elements. This model leads to $\overline{\Delta^{2}}=L^{2}\left(t u_{r m s} / L\right)^{\gamma}$ where $\Delta$ is the separation between fluid element pairs, the overbar symbolizes an average over many pairs and/or many flow realizations, $u_{r m s}$ is the r.m.s. turbulence velocity, $t$ spans an intermediate range of times bounded from above by the Lagrangian correlation time scale, and the Richardson exponent $\gamma$ is given by

$$
\gamma=\frac{2 d}{D_{s}} .
$$

In the Kraichnan model, the power spectrum and the structure functions of the advected scalar turn out to be power-law shaped with scaling exponents that are functions of the fractal co-dimension $D\left(=D_{s} / d\right)$ (Falkovich et al. 2001).

The two models of turbulent diffusion just mentioned differ in one important respect. The velocity field in the Kraichnan model is delta correlated in time whereas stagnation points in isotropic turbulence have been shown to be statistically persistent in the sense of being long-lived and slow moving (Goto et al. 2005 and Osborne et al. 2006). By finding a way to position stagnation points at will in a laboratory flow we hope to obtain a way to design a continuous power-law shaped energy spectrum with a chosen exponent $p$ determined by $D_{s}$. By then controlling the time dependence of this laboratory flow, we can hope to realize in the laboratory either the Kraichnan model of turbulent diffusion (in the case where the time dependence is such that the flow is effectively delta-correlated in time) or a situation where pair diffusion and concentration fluctuation statistics are determined by stagnation points and their spatial distribution (in the case where the time dependence is such that stagnation points are statistically persistent). In this way we should be able to obtain knowledge about the relations between spatio-temporal flow structure (multi-scale streamline topology and its time dependence) and scalar diffusion. Such relations cannot be obtained directly from turbulent flows where the spatio-temporal flow structure is uncontrolled and not fully understood. We will also be able to study the dependence of scalar variance decay and mixing (mixing being the result of combined stirring and molecular diffusion) on spatio-temporal flow structure (whether vortical, chaotic or multi-scale in space; see Vassilicos 2002 for a review).

Usually, eddies mix contaminants and scalar concentrations over scales comparable to their own size. Such mixing is therefore of limited applicability and perhaps also limited efficiency, whereas mixing by multi-scale forcing targets kinetic energy at various specific scales which can be chosen so as to maximize mixing efficiency. Following this line of thought, we might find flows with perhaps unusual mixing properties because some of the multi-scale flows that we generate in the laboratory by controlling a wide range of scales are in fact laminar. Laminar multi-scale velocity fields are a new concept and might turn out to be efficient mixers if they require little power to be run and have turbulent-like mixing properties. A similar concept of effective mixers exists in relation to chaotic advection where two-dimensional single-scale but timedependent velocity fields or three-dimensional velocity fields with chaotic streamlines (whether time-dependent or not) generate multi-scale scalar fields but without the velocity field necessarily being multi-scale in space (see Ottino 1989). 


\subsection{Turbulent-like flow simulations in the laboratory}

In this work we seek to generate quasi-two-dimensional flows with a multi-scale 'cat's eyes within cat's eyes' streamline structure such as in figure 1 and with the possibility to modify this fractal streamline structure and its fractal dimension $D_{s}$ (defined in (1.2)) at will. We propose to achieve this by fractal electromagnetic forcing.

Numerous previous works have used electromagnetic (EM) forcing to generate turbulent and chaotic quasi-two-dimensional flows (e.g. Sommeria 1986; Dolzhanskii et al. 1992; Cardoso, Marteau \& Tabeling 1994; Paret et al. 1997; Williams, Marteau \& Gollub 1997; Voth, Haller \& Gollub 2002; Rothstein, Henry \& Gollub 1999; Boffeta et al. 2005). Multi-scale forcing has been applied by Queiros-Conde \& Vassilicos (2001), Staicu et al. (2003) and finally Hurst \& Vassilicos (2006) who used fractal grids to stir the flow over many scales at once (see also the DNS of Mazzi \& Vassilicos 2004 and Biferale, Lanotte \& Toschi 2004). Here we combine both approaches to create a multi-scale fractal EM forcing of a quasi-two-dimensional flow in the laboratory.

In this paper we try to answer the following questions:

(i) Is it possible to generate a controlled quasi-two-dimensional multi-scale flow with a cat's eyes within cat's eyes flow topology in the laboratory?

(ii) Is the energy spectrum of such a flow continuous and power-law shaped and controlled by the multi-scale distribution of forced stagnation points?

(iii) What are the stirring properties of such a flow?

\section{Electromagnetically fractal forced thin layer of brine}

\subsection{Experimental set-up and fractal EM forcing}

A schematic of our rig is shown in figure $2(a)$. The brine flow is activated by EM forcing (Lorentz body forces $\boldsymbol{j} \times \boldsymbol{B}$ where $\boldsymbol{j}$ is the electric current density and $\boldsymbol{B}$ is the magnetic field) produced by permanent magnets $(\boldsymbol{B}$, Bonded $\mathrm{NdFeB}, B r \simeq 0.68 T$ ) placed under the (horizontal) brine-supporting wall, and electric currents generated by platinum electrodes on opposite sides of the tank as shown in figure 2(a) (there are, on each side, 43 electrodes of the same potential with a typical spacing of about $4 \mathrm{~cm}$ between them, and the two sides have opposite polarities). Each electrode is made of 16 platinum wires of $40 \mathrm{~mm}$ length and $11.5 \mu \mathrm{m}$ diameter. A resistance of $20 \Omega( \pm 0.1 \%)$ is added to balance and control the electric current in each electrode.

We keep in the regime where the electric field $\boldsymbol{E}$ imposed by the working electrodes is strong compared to the induced electric field $\boldsymbol{u} \times \boldsymbol{B}$ (the flow velocities $\boldsymbol{u}$ and the magnetic field $\boldsymbol{B}$ have small enough magnitudes as we verify in §2.3). Hence, the electric current density is fully controlled by the imposed electric field:

$$
\boldsymbol{j}=\sigma(\boldsymbol{E}+\boldsymbol{u} \times \boldsymbol{B}) \rightsquigarrow \boldsymbol{j} \simeq \sigma \boldsymbol{E}
$$

where $\sigma$ is the electrical conductivity. It has been verified in the experiment that the electric field is almost uniform across the horizontal square area measuring $1.3 \mathrm{~m} \times 1.3 \mathrm{~m}$ which is centred at the stagnation point between the two largest magnets (the magnet set-up is described below). This square area covers, and is in fact much larger than, the entire magnet set-up.

We also keep in the regime where magnetic Reynolds numbers are very small, i.e. $\mu \sigma u l \ll 1$ where $\mu$ is the magnetic permeability $\left(\mu \approx \mu_{0}=4 \pi \times 10^{-7} \mathrm{VsA}^{-1} \mathrm{~m}^{-1}\right)$ and $u$ and $l$ represent a range of flow velocities and length scales characterizing each scale iteration in the fractal-like flow structure that we want to design. Being generated by permanent magnets, the magnetic field is stationary in time and the induction 
(a)

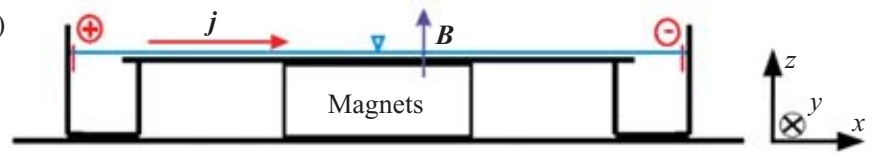

(b)

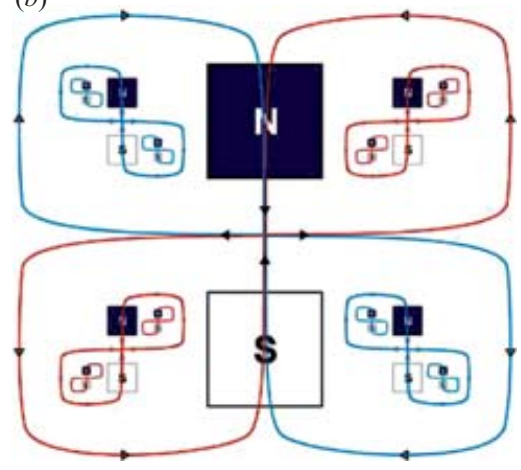

$(d)$

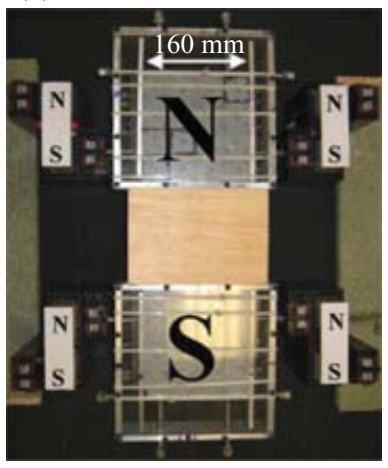

(c)

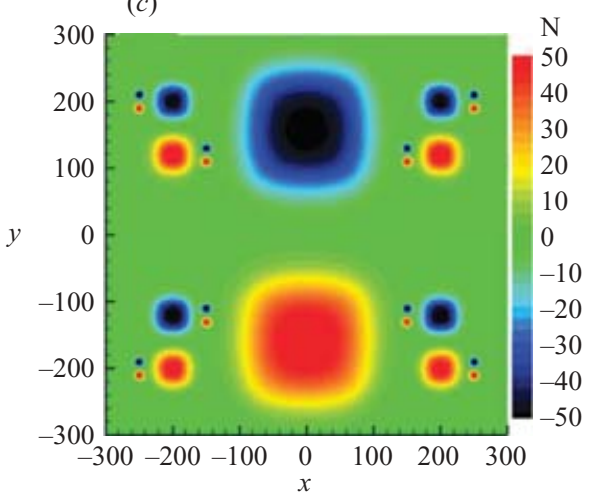

(e)

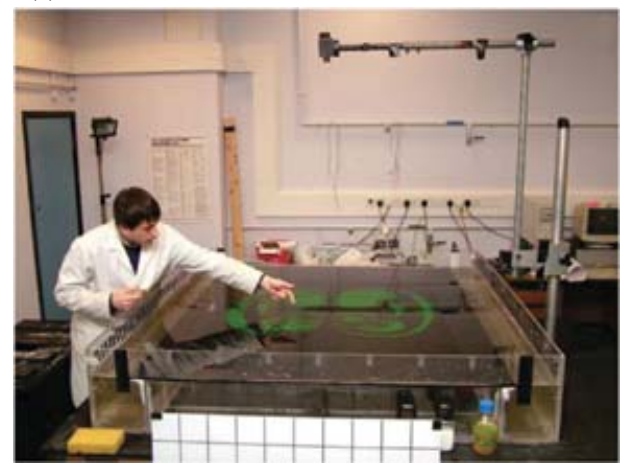

FIgURE 2. (a) Rig's schematic for electromagnetic forcing of a shallow brine layer. $(b)$ Schematic of a fractal flow and associated permanent magnets. (c) Electromagnetic forcing distribution computed with $I=1 \mathrm{~A}, B_{r e f}=1 \mathrm{~T} ; f_{y}$ in $\mathrm{N} \mathrm{m}^{-3} ; x$ and $y$ in $\mathrm{mm}$. (d) Under-wall distribution of permanent magnets used in experiments. $(e)$ The rig.

equation reduces to a Laplace equation:

$$
\frac{\partial \boldsymbol{B}}{\partial t}=\operatorname{curl}(\boldsymbol{u} \times \boldsymbol{B})+\frac{1}{\mu \sigma} \nabla^{2} \boldsymbol{B} \leadsto \nabla^{2} \boldsymbol{B} \simeq 0 .
$$

The Lorentz force field $\boldsymbol{j} \times \boldsymbol{B}$ is therefore perfectly controlled and independent of the flow (which is a special case of magnetohydrodynamics where, in general, the velocity, magnetic and electric fields are coupled, see Moreau 1991; Davidson 2001). Here, our control parameter is the electric current $I$ which equals the uniform electric current density $|\boldsymbol{j}|$ times the brine's vertical cross-sectional area parallel to a side of the square tank. In this paper we keep $I$ constant in time so as to generate time-independent forcing; of course this can, and will, be relaxed in future works.

To design a multi-scale 'cat's eyes within cat's eyes' streamline pattern similar to the one observed in two-dimensional turbulence by Fung \& Vassilicos (1998) and Goto \& Vassilicos (2004) (see figure $2 b$ ), the applied EM forcing must be fractal-like. The electrical current density $(\boldsymbol{j})$ being uniform, the spatial distribution of the EM forcing 
is determined by the positions and sizes of the magnets (see figure $2 b$ ). Figure $2(d$ ) shows the positions and sizes of our magnets (the brine-supporting wall is placed above them). Three scales of EM forcing are applied relating to three horizontal sizes of square magnets: $l_{0}=160 \mathrm{~mm}$ (M160 magnets), $l_{1}=40 \mathrm{~mm}$ (M40 magnets), $l_{2}=10 \mathrm{~mm}$ (M10 magnets). The vertical sizes of the magnets are $60 \mathrm{~mm}, 40 \mathrm{~mm}$ and $10 \mathrm{~mm}$, respectively. The size of the tank $\left(1700 \times 1700 \mathrm{~mm}^{2}\right)$ is large compared to the size of the magnets and the EM forcing area represents only $2.8 \%$ of the area of the brine-supporting wall. This percentage is small compared to all previous similar experimental set-ups (e.g. Cardoso et al. 1994; Williams et al. 1997). Each forcing scale is made of a pair of north $(\mathrm{N})$ and $\operatorname{south}(\mathrm{S})$ magnets (see figure $2 d$ ) placed on a horizontal iron plate under the brine-supporting wall.

The pair-spacing is about the horizontal size of the associated magnets, and the iron plates (supporting each magnet-pair) are of sufficient thickness to close the magnetic field.

The main point of this design is to generate and control opposite forces, due to the $\mathrm{N}-\mathrm{S}$ magnet pairs, so as to create and control flow stagnation points for each scale of forcing. The number and positions of flow stagnation points at each scale depend on the number and positions of magnet pairs at these scales. The total number of flow stagnation points depends on the fractal-like organization of the magnet-pairs which we now describe.

The geometry of the EM forcing can be described in terms of iterative relations for the coordinates $\left(x_{n}, y_{n}\right)$ of the centres of $\mathrm{N}-\mathrm{S}$ pairs of magnets of horizontal size $l_{n}(n=0,1,2)$ and the coordinates $\left(x_{N n}, y_{N n}\right)$ and $\left(x_{S n}, y_{S n}\right)$ of the $\mathrm{N}$ and $\mathrm{S}$ magnets in a pair. These iterative relations for the distribution of magnet-pairs in figure 2 are:

$$
\begin{aligned}
& l_{n+1}=\left(\frac{1}{R}\right) l_{n}, \\
& y_{n+1}=y_{n} \pm l_{n}, \\
& x_{n+1}=x_{n} \pm(1+1 / R) l_{n},
\end{aligned}
$$

from which only those points obeying $x_{n}= \pm(1+1 / R) y_{n}$ are kept, and

$$
\begin{aligned}
& y_{N n}=y_{n}+l_{n}, \\
& y_{S n}=y_{n}-l_{n}, \\
& x_{N n}=x_{S n}=x_{n} .
\end{aligned}
$$

In our case the aspect ratio between two consecutive scales of forcing is $R=4$ and $x_{0}=y_{0}=0$ which is at the centre of the tank.

The distance of each magnet pair from the brine supporting wall is adjusted so as to make the Lorentz body forces about equal above each magnet pair in the present runs of our experiment. It is possible to modify the forcing balance between scales (effectively the power spectrum of the EM forcing) without changing the geometry of the forcing, but we leave this exercise for future work. The EM forces can be computed by using the method described in Rossi (2001) and Akoun \& Yonnet (1984), but we need to know the thickness $H$ of the layer of brine to make this computation meaningful and use it to determine the distances of magnet pairs from the wet side of the brine supporting wall. We explain how we decide on the value of 
$H$ in $\S 2.2$. At the end of $\S 2.2$, we determine these distances and compute the power spectrum of our EM forcing.

\subsection{Brine layer's salt concentration and thickness and quasi-two-dimensionality of horizontal flow}

A number of previous studies (e.g. Williams et al. 1997; Julien, Paret \& Tabeling 1999) used two superimposed layers of brine with two different salt concentrations (assumed homogeneous) so as to improve the quasi-two-dimensionality of the flow. Other previous studies used one single layer of brine (e.g. Cardoso et al. 1994).

Two superimposed layers of brine with different homogeneous salt concentrations at ambient temperature would give rise within less than 20 min to a mixed layer of about $1 \mathrm{~mm}$ between them because of molecular diffusion (the molecular diffusivity of salt in water is about $10^{-9} \mathrm{~m}^{2} \mathrm{~s}^{-1}$ at a temperature of $15^{\circ} \mathrm{C}$ ). In addition, the process of stratification, which is faster than molecular diffusion, would cause the vertical profile of salt concentration to change with time. The salt concentration is a determining factor of the brine's electrical conductivity $\sigma$. Its variation with time and depth allied with the magnetic field's exponential decrease with vertical distance from the magnets (scaled by their size) would cause uncontrolled variations in the EM forcing. For all these reasons, and because our layer of brine must be small compared to the size of our smallest magnets (which size determines the extent of their magnetic field's strength), a double layer of brine is not appropriate for our purposes, particularly because of our need for long time measurements and because of the large size of our tank which prohibits regular and fast refilling. Hence, we have opted for a single layer of brine with stable time-independent stratification.

An immediate implication is that, in order to minimize vertical gradients of EM forcing within the stratified layer of brine, the brine must have a high concentration of salt. This is because a high concentration of salt gives a very small dependence of conductivity on salt concentration compared to the usual concentrations in seawater: $\partial \sigma / \partial C \simeq 0.0656\left(\mathrm{~S} \mathrm{~m}^{-1}\right) /\left(\mathrm{gl}^{-1}\right)$ for a salt concentration of about $158 \mathrm{gl}^{-1}$, which is what we have chosen, compared to usual salt/sea-water $\left(35 \mathrm{gl}^{-1}\right)$ where $\partial \sigma / \partial C \simeq$ $1.3\left(\mathrm{~S} \mathrm{~m}^{-1}\right) /\left(\mathrm{gl}^{-1}\right)$. It is not possible to produce brine with salt concentrations much larger than what we have chosen because the salt then clumps together and precipitates. Our value of $\sigma$ is about $16.6 \mathrm{~S} \mathrm{~m}^{-1}$; also, the mass density of our brine is $10 \%$ higher than that of fresh water.

The thickness $H$ of our single layer of brine must be smaller than our smallest magnet size $(10 \mathrm{~mm})$ because the magnetic field weakens exponentially with distance from the magnet according to the magnet's size. Hence, $H=5 \mathrm{~mm}$ fulfils this condition. However, the thickness $H$ must also be small enough to inhibit as much as possible three-dimensionality of flow in case of strong EM forcing, but large enough for the bottom friction not to bring the flow to near standstill away from the magnets. To quantify these conditions, we refer to the momentum equation for the flow of our electromagnetically forced layer of brine which includes gravity, pressure, viscous and Lorentz body forces as follows

$$
\frac{\mathrm{D} \boldsymbol{u}}{\mathrm{D} t}+\nabla P / \rho-\boldsymbol{g}=v \nabla^{2} \boldsymbol{u}+\boldsymbol{f}
$$

where $\mathrm{D} \boldsymbol{u} / \mathrm{D} t$ is the convective derivative of the velocity field $\boldsymbol{u}, P$ denotes the pressure field, $\rho$ the mass density of our brine (assumed constant in space and time for the incompressible flows considered here), $g$ the acceleration due to gravity, $v$ the kinematic viscosity of our brine (about $1.326 \times 10^{-6} \mathrm{~m}^{2} \mathrm{~s}^{-1}$ ), and $\boldsymbol{f} \equiv \boldsymbol{j} \times \boldsymbol{B} / \rho$. Of 
importance also is the incompressibility condition

$$
\operatorname{div} \boldsymbol{u}=0 .
$$

For the Lorentz forces to be able to overcome viscous forces (including, most importantly, the bottom friction), the Hartmann number must be greater than 1 . This number is defined here as $H_{a}^{2} \equiv f_{r m s} / v\left(u_{r m s} / H^{2}\right)$, where $f_{r m s}$ is the root mean square of a characteristic magnitude of $\boldsymbol{f}$ which we specify in $\S 2.3$ and $u_{r m s}$ is the root mean square horizontal fluid velocity at the free surface of the brine; the average is taken over the horizontal square area measuring $80 \mathrm{~cm} \times 80 \mathrm{~cm}$, containing all the magnets and centred at the stagnation point between the two largest magnets.

For the flow to be as quasi-two-dimensional as possible, the ratio of $H$ to the size of the tank must be very small which is indeed the case if $H$ is taken to be about half the scale of the smallest magnets, i.e. $H=5 \mathrm{~mm}$. Following the work of Satijn et al. (2001), a better test of quasi-two-dimensionality of horizontal vortical flow is based on the idea that the vortex pressure pumping should be smaller than the viscous damping, a condition which can be expressed by the requirement that the pressure gradient term in (2.3), which we might scale as $u_{r m s} / H$ should be smaller than the viscous term which might be expected to have a scaling bounded from above by $v u_{r m s} / H^{2}$. In terms of the Reynolds number $R e_{3 D}=u_{r m s} H / v$, the condition of Satijn et al. (2001) therefore requires that $\operatorname{Re}_{3 D}$ should not be too large. A complementary condition for quasi-two-dimensionality is that the Froude numbers $u / \sqrt{g l}$ should all be smaller than 1 so as to avoid pressure differences comparable to $\rho g$ across horizontal distances $l$ ranging between $H$ and $l_{0}$ (the largest magnet size) with corresponding characteristic velocities $u$. These Froude numbers are all bounded from above by $F r \equiv u_{r m s} / \sqrt{g H}$ and it is this $F r$ which we require to be much smaller than 1 in our experiment $(0.004 \leqslant F r \leqslant 0.073)$. In $\S 3.2$, we verify quasi-two-dimensionality using our velocity field measurements and the criteria of Satijn et al. (2001) plotted in figure 7. A rough verification of quasi-two-dimensionality was also achieved by using the free surface as a mirror and checking, for a broad range of electrical currents, that images of parallel lines of reflected light stay parallel and clear (no blurring effects) and are not deformed during fluid motion. In fact, we have explored intensities from 0 to $3 \mathrm{~A}$ as well as various brine thicknesses $(2.5 \mathrm{~mm}$ to $10.6 \mathrm{~mm})$ to check the sensibility of our experiment (three-dimensionality, confinement, topology) to these parameters. All our experimental runs have been carried out for quasi-twodimensional flows on the basis of our free-surface/optical criterion and the criteria of Satijn et al. (2001).

In $\S 3.2$ and at the end of $\S 3.1$, we report a posteriori verifications that the horizontal flow at the free surface of the brine layer is also effectively incompressible, which means that the vertical gradient of the vertical flow velocity at the free surface is negligible compared to the other terms in (2.4).

We experimented with various values of $H$ and eventually settled on $H=5 \mathrm{~mm}$. Detailed thickness measurements over the entire brine supporting wall were carried out before each experimental session. Averaged over many experimental sessions and over many stations on the brine supporting wall, our brine layer's mean thickness is in fact $H_{\text {mean }}=5.028 \mathrm{~mm}$ with standard deviation equal to $H_{r m s}=0.029 \mathrm{~mm}$. For a given experimental session, the typical standard deviation of the layer's thickness due to wall/measurement imperfections is about $0.153 \mathrm{~mm}$. The decrease of brine thickness by evaporation is about $0.3 \mathrm{~mm} /$ day which translates into a loss of less than $0.1 \mathrm{~mm}$ during a typical experimental session. The brine is left to rest before each experimental session so as to reach a naturally stratified shallow layer. The final 


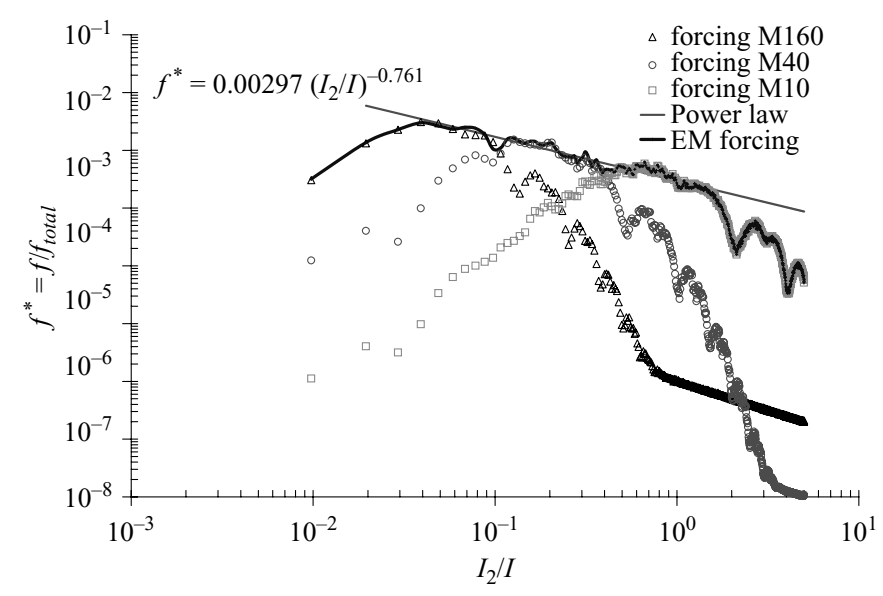

FIGURE 3. Spectrum of the electromagnetic forcing. Symbols represent the forcing associated to the different sizes of magnets: $\triangle$, for M160; , for M40; $\square$, for M10. Lines represent the total forcing and its power-law interpolation.

thickness adjustment to compensate for evaporation is done with fresh water. This leads to an initial $0.3 \mathrm{~mm}$ thickness of fresh water on top of the brine layer which quickly mixes with the brine.

Given our magnets and their positions, given the thickness and electrical conductivity of our brine layer and given an imposed potential between the two sets of electrodes, the fractal EM force field $f$ in (2.3), which drives the flow, is computed three-dimensionally using the method described in Rossi (2001) and Akoun \& Yonnet (1984). This computer calculation gives, for example, $(1 / H) \int_{0}^{H} f(x, y, z) \mathrm{d} z$ where $x, y$ are horizontal coordinates and $z$ is the vertical coordinate. Figure $2 c$ shows a distribution of calculated values of $(1 / H) \int_{0}^{H} f(x, y, z) \mathrm{d} z$ in the plane $(x, y)$. Local mean values of the forcing above each magnet are then calculated by averaging $(1 / H) \int_{0}^{H} f(x, y, z) \mathrm{d} z$ over the horizontal $x, y$ area of the magnet, and they are used to adjust the distance of each magnet from the brine supporting wall. These local mean values are $f_{40}=1.025 f_{160}$ and $f_{10}=1.094 f_{160}$ for the M40 and M10 magnets in terms of the local mean forcing $f_{160}$ of M160 magnets when their distances from the brine supporting wall are $-40 \mathrm{~mm}$ for M160, $-11.2 \mathrm{~mm}$ for M40 and $-1 \mathrm{~mm}$ for M10. Thus the forcing is kept nearly constant across length scales of EM forcing with, however, a slight reinforcement of the smaller scales so as to make the fractal forcing a little more robust in view of the fact that the three-dimensional positions of the smallest magnets are the most delicate to adjust.

A more instructive quantity might be the power spectrum of $(1 / H) \int_{0}^{H} \boldsymbol{f}(x, y, z) \mathrm{d} z$ which we have also calculated and plot in figure 3. This plot shows that the power spectrum of our fractal EM forcing is continuous and power-law shaped over almost two decades of wavenumber (with an exponent of about $-0.761 \approx-3 / 4$ ) even though, as figure 2(c) makes clear, the forcing acts on a discrete number of length scales. In figure 3, we also plot the power spectra of each elementary set of magnet pairs: the one pair of M160 magnets, the four pairs of M40 magnets and the eight pairs of M10 magnets. These power spectra make it clear that each scale of EM forcing has its own non-power-law spectral signature and that they combine together to form the power-law spectrum of the entire fractal EM forcing. At a given wavenumber, the 


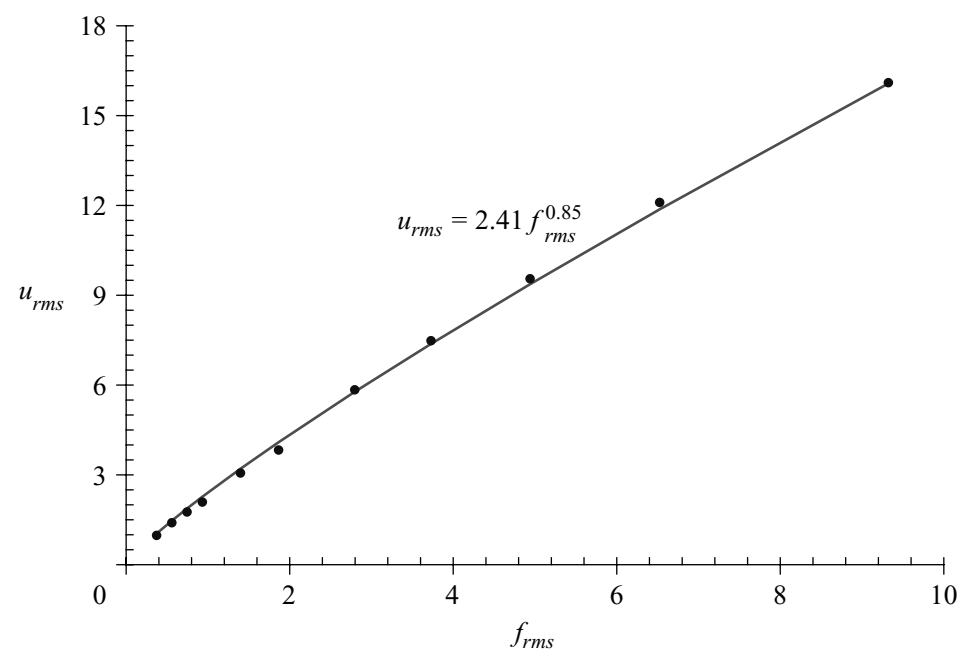

FIGURE 4. Evolution of the flow intensity, $u_{r m s}$, with the intensity of the forcing, $f_{r m s}$.

forcing is dominated by the magnets whose length-scale corresponds most closely to that wavenumber.

\subsection{Intensity of the zero-mean horizontal velocity field and values of the dimensionless parameters}

In summary, the range of electric currents (which control the range of $u_{r m s}$ ) used in this experiment must ensure that the root mean square horizontal flow velocity $u_{r m s}$ satisfies $H_{a}^{2}>1, R_{3 D}$ not too large, $R e_{m} \equiv \mu \sigma u_{r m s} l_{0} \ll 1$ and $|\boldsymbol{E}| \gg|\boldsymbol{u} \times \boldsymbol{B}|$. The mean horizontal flow velocity averaged over the horizontal $80 \mathrm{~cm} \times 80 \mathrm{~cm}$ square area containing all the magnets and centred at the stagnation point between the two largest magnets can be expected to be zero and does indeed turn out to be so.

The horizontal velocity field generated by the imposed electrical potential in the laboratory experiment is measured using particle image velocimetry (PIV) which we describe in $\S 3$. Anticipating this description, we plot in figure 4 the dependence of the measured $u_{r m s}$ on the calculated $f_{r m s}$ (the root mean square of $(1 / H) \int_{0}^{H} f(x, y, z) \mathrm{d} z$ where the mean is calculated over the $x, y$ averaging area specified in the previous paragraph) which shows that $u_{r m s} \sim f_{r m s}^{0.85}$ for electrical currents up to about $1 \mathrm{~A}$, and we report in table 1 that, for such currents, the dimensionless parameters $H_{a}^{2}$ and $R e_{3 D}$ do take values within the bounds required. The condition $R e_{m} \ll 1$ is also met as $R e_{m}$ is of order $10^{-9}$ and so is $|\boldsymbol{E}| \gg|\boldsymbol{u} \times \boldsymbol{B}|$ everywhere as $\left(u_{\max } B_{r} / 2\right) /|\boldsymbol{E}|$ is smaller than 0.01 for all cases of electric currents tried and documented here $\left(u_{\max }\right.$ is the maximum fluid velocity magnitude in the $(x, y)$-plane of measurements, see table 1 , and $B_{r} / 2 \approx 0.34 \mathrm{~T}$ is the value of the magnetic field at the surface of each magnet (in case of no interference from the magnet's opposite surface) which is therefore an upper bound for $|\boldsymbol{B}|$ anywhere in the flow). Table 1 also includes values of the Eulerian integral length scale $L_{E}$ (obtained from PIV measurements, see $\S \S 3$ and 4) of the horizontal flow field and of another Reynolds number, $R e_{2 D} \equiv u_{r m s} L_{E} / \nu$.

The power law $u_{r m s} \sim f_{r m s}^{0.85}$ corresponds to a force balance which is mostly one between viscous (predominantly bottom friction) and electromagnetic forces with some influence, nevertheless, of inertial forces. Indeed, as argued by Thibault \& Rossi (2003) and as might be expected by simple inspection of (2.3), a purely viscous balance would lead to $u_{r m s} \sim f_{r m s}$ whereas a purely inertial balance would lead to 


\begin{tabular}{lcccccrr}
\hline$I(\mathrm{~A})$ & $u_{r m s}\left(\mathrm{~mm} \mathrm{~s}^{-1}\right)$ & $u_{\max }\left(\mathrm{mm} \mathrm{s}^{-1}\right)$ & $L_{E}(\mathrm{~mm})$ & $f_{r m s}\left(\mathrm{~N} \mathrm{~mm}^{-3}\right)$ & $H_{a}^{2}$ & $\operatorname{Re}_{2 D}$ & $\operatorname{Re}_{3 D}$ \\
0.04 & 0.977 & 3.1 & 156.1 & 0.373 & 7.11 & 600 & 3.7 \\
0.06 & 1.40 & 4.5 & 155.9 & 0.559 & 7.49 & 860 & 5.3 \\
0.08 & 1.76 & 5.3 & 157.5 & 0.746 & 8 & 1100 & 6.6 \\
0.1 & 2.09 & 6.2 & 158.6 & 0.932 & 8.11 & 1300 & 7.9 \\
0.15 & 3.06 & 8.6 & 162 & 1.40 & 8.66 & 1900 & 11.5 \\
0.2 & 3.83 & 11.1 & 165.1 & 1.86 & 9.17 & 2300 & 14.4 \\
0.3 & 5.84 & 15.6 & 172.2 & 2.80 & 9.02 & 3600 & 22 \\
0.4 & 7.48 & 19.9 & 177.8 & 3.73 & 9.33 & 4600 & 28.2 \\
0.53 & 9.55 & 25 & 183.1 & 4.94 & 9.61 & 5900 & 36 \\
0.7 & 12.1 & 31 & 189.3 & 6.53 & 9.89 & 7400 & 45.6 \\
1 & 16.1 & 40 & 195.3 & 9.32 & 10.5 & 9900 & 60.7
\end{tabular}

TABLE 1. Typical scales of the multi-scale flow according to the forcing intensity.

$u_{r m s} \sim f_{r m s}^{1 / 2}$. (For comparison, recall that the inertial transfer terms were inhibited in the fractal-forced DNS turbulence of Mazzi \& Vassilicos 2004.)

\section{Flow measurements}

\subsection{Visualization and particle image velocimetry $(P I V)$}

The measurements presented in this paper are based on visualization, using both dye and PIV. The light is controlled by the positions of two $500 \mathrm{~W}$ projectors on the sides of the tank.

For dye visualization, a standard digital camera with $6 \times 10^{6}$ pixels has been used. The main dye used is fluorescein. For multi-colour visualizations, red and white screen-printing water-based inks were also used.

For our PIV measurements, we used a camera with a resolution of $2048 \times 2048$ pixels and a dynamic range of $64 \mathrm{db} 12$ bit (Kodak MegaPlus Model ES 4.0). The PIV software is an in-house software of the Department of Mechanical Engineering of Imperial College (Kolokotronis 2006). This PIV software is based on the calculation of cross-correlations and allows for a choice of correlation window sizes and displacements 'pixel by pixel' without loss of accuracy. We have optimized the size of the correlation window to ensure good tracking for large displacements as well as high spatial resolution. Two measurement campaigns have been carried out: one within the physical frame of $80 \mathrm{~cm} \times 80 \mathrm{~cm}$ centred at the stagnation point between the largest magnets and the other within the bottom right-hand quarter of that frame which measures $40 \mathrm{~cm} \times 40 \mathrm{~cm}$.

(i) For the $80 \mathrm{~cm}$ frame (the exact size of this frame is $L_{P I V}=813.4 \mathrm{~mm}$ ), the correlation windows have $16 \times 16$ pixels (i.e. this window's area is $6.35^{2} \mathrm{~mm}^{2}$ ), the search window have $42 \times 42$ pixels, and therefore the maximum displacement is of 13 pixels, and the overlap in each direction is of 9 pixels. This leads to a measurement grid of $287 \times 287$ velocity vectors. The physical length of one pixel is about $0.3972 \mathrm{~mm}$. This resolution gives about 13 velocity vectors above the smallest magnets.

(ii) For the $40 \mathrm{~cm}$ frame $\left(L_{P I V}=413.8 \mathrm{~mm}\right)$, the correlation windows have $20 \times 20$ pixels (i.e. $4^{2} \mathrm{~mm}^{2}$ ), the search window have $52 \times 52$ pixels, so the maximum displacement is of 16 pixels, and the overlap is of 13 pixels. This leads to a measurement grid of $285 \times 285$ velocity vectors. The physical length of one pixel is about $0.2020 \mathrm{~mm}$. This resolution gives about 50 velocity vectors above the smallest magnets. 

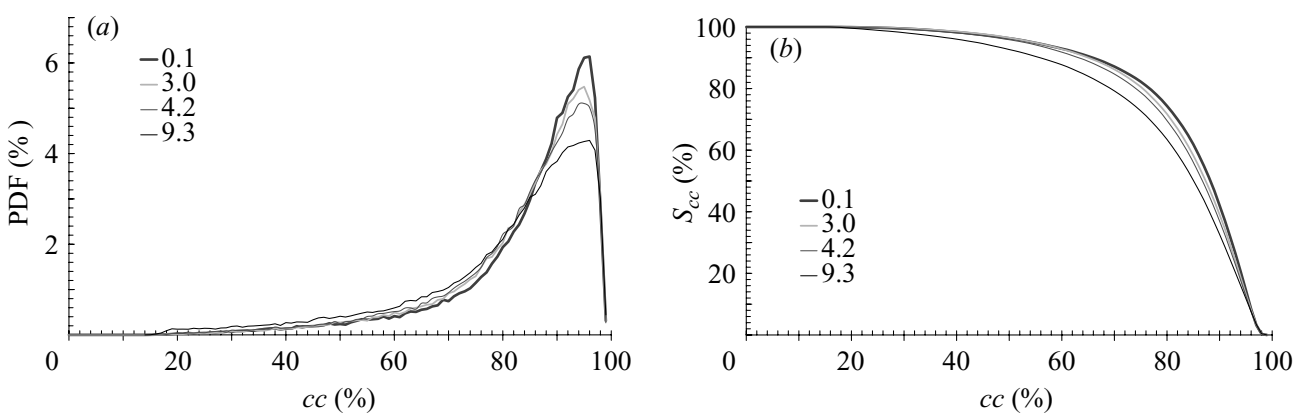

FiguRE 5. Distribution of correlation coefficients, $c c$, at different dimensionless times $t^{*}=$ $t /\left(L_{E} / u_{r m s}\right)$ with $L_{E} / u_{r m s}=29.49 \mathrm{~s}$, for the case $I=0.3 \mathrm{~A}$, frame $80 \mathrm{~cm}$. (a) Probability density function $(\mathrm{PDF})$ in $\%$. (b) $S_{c c}=1-\int_{0}^{c c} \operatorname{PDF}(c) \mathrm{d} c$ in $\%$.

The particles used for PIV are Chemingum P83 (white powder), with a range of scales between about 100 to $600 \mu \mathrm{m}$ and a density of 1.03 compared to fresh water. Soap is added to the water to reduce capillarity effects. Great care has been taken in comparing dye evolution and particle trajectories in various test runs to check their agreement. These observations include, for example, tests where we have checked that particles placed in clusters spread and split as a result of the flow in very good agreement with the spreading of the dye.

PIV measurements are initialized with a homogeneous and dense star-field (without clusters) where particles are present in all the correlation windows. The cut-off for the value of the correlation coefficient (see Raffel, Willert \& Kompenhans 1998) is chosen at the unusually high value of $66 \%$ to ensure quality. In figure 5, we plot the probability density function (PDF) of the correlation coefficient $(c c)$ as well as $S_{c c}=1-\int_{0}^{c c} \operatorname{PDF}(c) \mathrm{d} c$ for various times of measurement, in the case of the $80 \mathrm{~cm}$ frame and with $I=0.3 \mathrm{~A}$. The quality of the cross-correlations is good as the peak of their PDF is at $95 \%$ and more than $90 \%$ of correlation coefficients (i.e. measurements points) are above the threshold of $66 \%$.

Even though the flow is laminar and two-dimensional, it is not a trivial flow for PIV as it is a multi-scale flow with multi-scale velocity gradients. These difficulties are dealt with by the high resolution of our PIV measurements.

We use the horizontal divergence of the horizontal velocity field to establish the sensitivity and accuracy of our PIV. We assume quasi-two-dimensionality of the horizontal flow (which we check independently in §3.2) which means that all non-zero values of this divergence are interpreted as resulting from PIV noise and mistakes. This way we obtain an estimation of our accuracy. We define the error $\Delta u$ on the velocity measurements of our PIV by

$$
\frac{\Delta u}{u_{\max }}=\frac{1}{1.633} \frac{\Delta_{x / y}(\operatorname{div} \boldsymbol{u})_{r m s}}{u_{\max }},
$$

where $u_{\max }$ is the maximum intensity of the velocity field, $\Delta_{x / y}$ is the length increment used to extract $\operatorname{div} \boldsymbol{u}$ from the PIV velocity field $\left(\Delta_{x / y}\right.$ is about the correlation window size), $(\operatorname{div} \boldsymbol{u})_{r m s}$ is the root mean square of the divergence field and 1.633 is the root mean square factor which takes into account all possible error additions caused by summing together the two derivative terms in $\operatorname{div} \boldsymbol{u}$.

In figure 6 , we plot $\Delta u / u_{\max }$ as a function of $\operatorname{Re}_{2 D}$ for different PIV fields. The higher quality of the $40 \mathrm{~cm}$ frame is due to a more accurate spatial resolution and to 


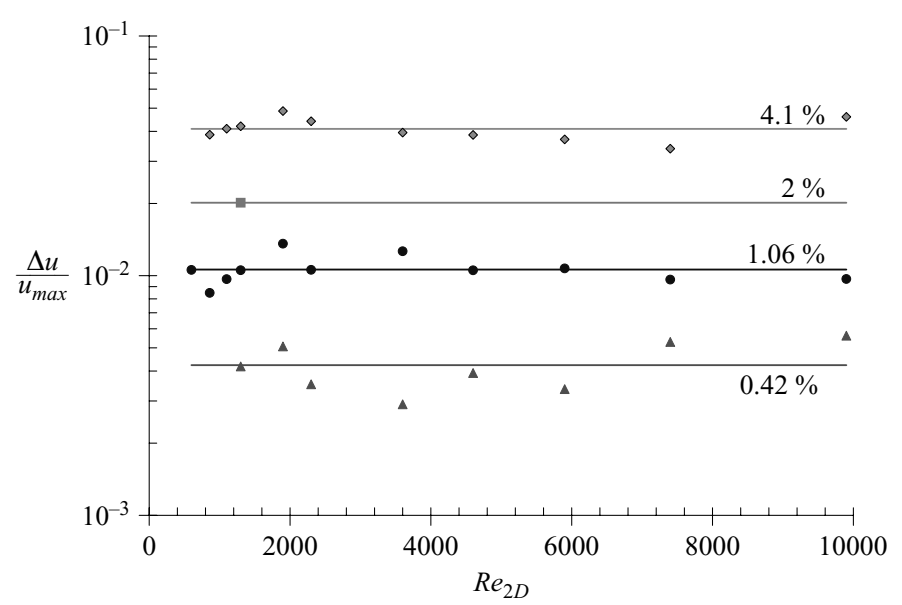

FIGURE 6. PIV sensitivity, $\Delta u$, normalized by the maximum velocity, $u_{\max }$ in function of the Reynolds number $R e_{2 D}$. $\bullet$, velocity fields for frame $80 \mathrm{~cm}$; $\mathbf{\square}$, velocity field with a $3 \times 3$ windows averaging for frame $80 \mathrm{~cm} ; \bullet$, mean velocity field for frame $80 \mathrm{~cm} ; \boldsymbol{\Lambda}$, mean velocity field for frame $40 \mathrm{~cm}$.

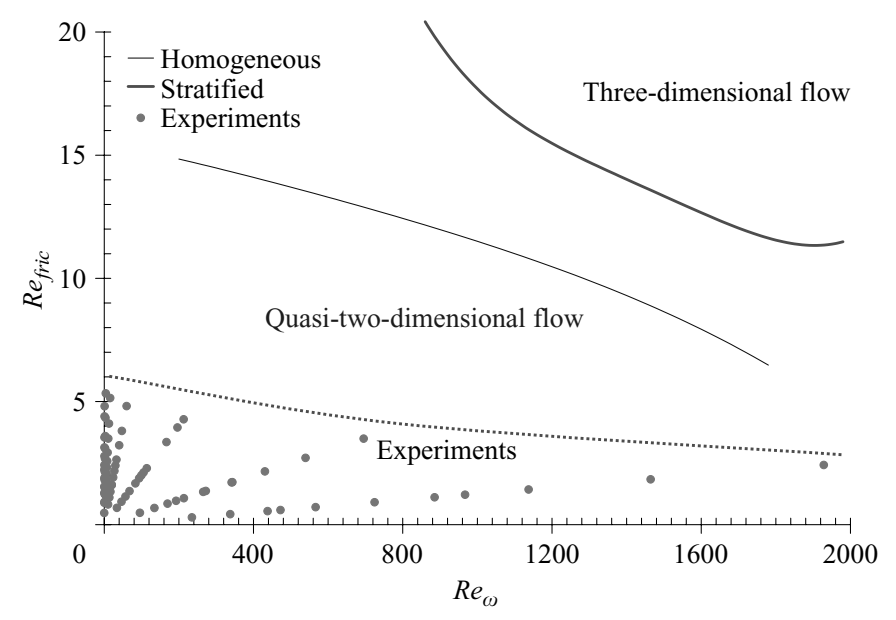

FIGURE 7. A posteriori analysis of quasi-two-dimensional aspects of the flow from PIV data. $R e_{\text {fric }}=(2 H)^{2} \omega / \pi^{2} v$ and $R e_{\omega}=\left(l_{e d}\right)^{2} \omega / v$. Lines represent the boundaries given by Satijn et al. (2001). represent the range of the experiments.

a larger maximum displacement which gives a better velocity resolution. The mean velocity fields in the quasi-stationary flow state are computed over 142 velocity fields (on average) and are used in our study of the multi-scale flow topology and our computations of energy spectra, see $\S \S 4$ and 5 . In the $80 \mathrm{~cm}$ frame, the mean value of $\Delta u / u_{\max }$ is about $1.06 \%$ whereas it is $0.4 \%$ in the $40 \mathrm{~cm}$ frame. The real-time measurements filtered with an average over $3 \times 3$ velocity grid points (recall that our windows overlap is of $\gtrsim 50 \%$ ) are used in our calculations of single fluid element and pair statistics, see $\S 6$. The corresponding $\Delta u / u_{\max }$ is about $2 \%$.

All these values lie within usual errors of good PIV measurement (for 13 pixels of maximum displacement) which is about $4.5 \%$. This confirms the good quality of our PIV measurements as this precision has only been achieved as a result of sub-pixel accuracy. 

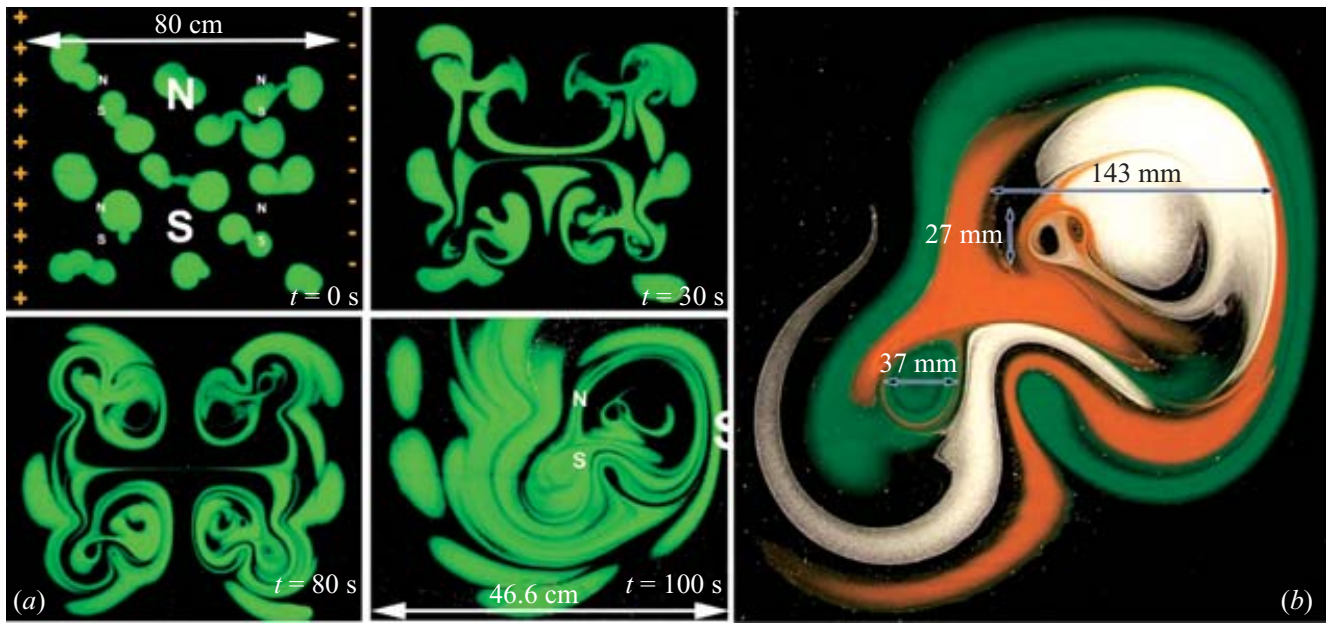

Figure 8. Dye visualizations for $I=0.3 \mathrm{~A},(a)$ Entire flow, magnets (M160 and M40) are indicated by $\mathrm{N}$ and $\mathrm{S}$, while the electrical potential is indicated by + and - . The power is switch ON at $t=0,(b)$ Quarter flow, picture taken about 75 s (i.e. $t\left(u_{r m s} / L_{E}\right)=2.5$ ) after switch $\mathrm{ON}$. Physical length scales are given on the pictures.
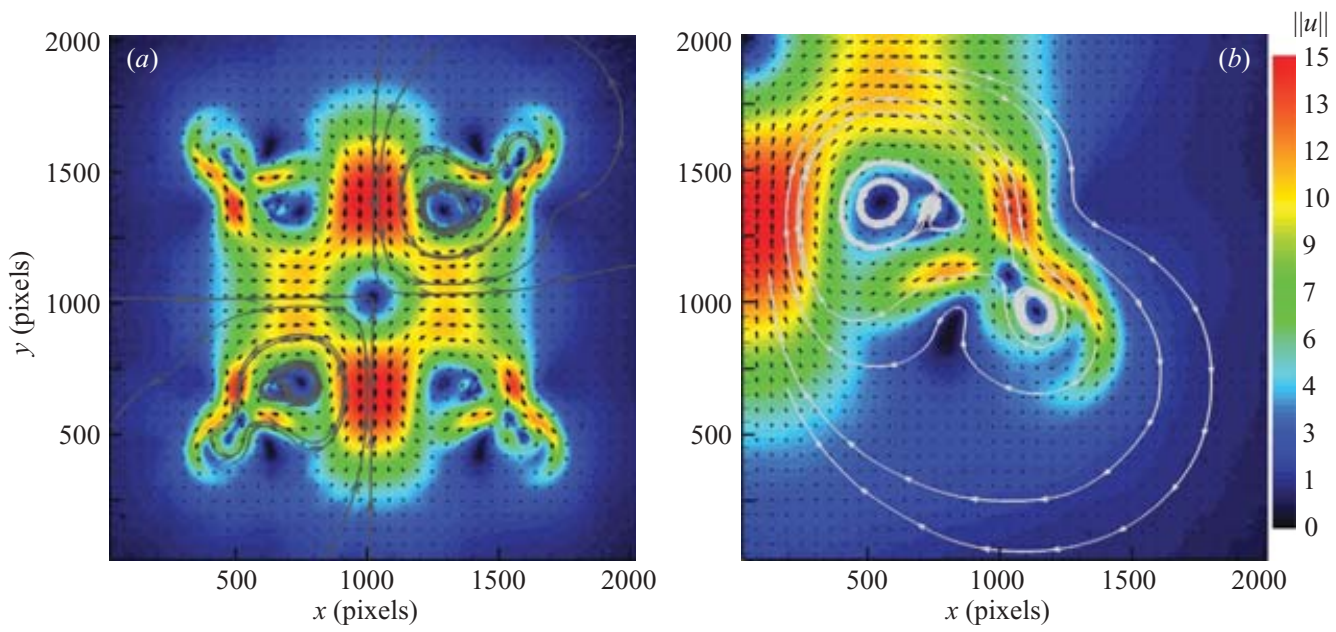

FiguRE 9. PIV measurements for $I=0.3 \mathrm{~A}:(a)$ entire flow, frame $80 \mathrm{~cm},(b)$ quarter of the flow, frame $40 \mathrm{~cm} .1$ arrow on 64 are represented, $\|u\|$ is the velocity intensity in $\mathrm{mm} \mathrm{s}^{-1}, x$ and $y$ in pixels. Some streamlines are given by grey and white lines.

In addition, the fact that $\Delta u / u_{\max }$ is within usual PIV noise shows that the non-zero values of the divergence do not come from extended three-dimensional flow effects of significant magnitude.

\subsection{Quasi-two-dimensional aspects}

Considering the large ratio of the tank size to $H$ and the relatively small values of $R_{3 D}$ above order 1 , the flow is expected to be quasi-two-dimensional without much influence from the bottom friction according to the criteria of Clercx et al. (2003) and Satijn et al. (2001).

We have checked the quasi-two-dimensionality of our flow a posteriori from PIV measurements analysis. Satijn et al. (2001) gives two boundary curves for a stratified 
and homogenous shallow layer of brine. Their criterion is based on the competition between the role of the bottom friction and the vorticity of a single vortex. The flow is thus considered as quasi-two-dimensional when the local energy present in the direction perpendicular to the wall is less than $1 \%$ of the energy present in planes parallels to the bottom wall. We use this criterion which is associated with the vorticity field analysed at different discrete scales to check finally that our flow is quasi-two-dimensional after PIV measurements. Figure 7 shows this criterion applied to experiments $(80 \mathrm{~cm}$ : $0.2 \mathrm{~A}$ to $1 \mathrm{~A})$ for $\omega_{r m s}$ (root mean square of vorticity) and $\omega_{r m s}+\sigma_{\omega_{r m s}}\left(\sigma_{\omega_{r m s}}\right.$ is the standard deviation of $\left.\omega_{r m s}\right)$ at different scales (from 14 pixels to 448 pixels). $R e_{\omega}$ represents the intensity of vortices, identified as an origin of three-dimensionality in the flow. $R e_{\text {fric }}$ represents the influence of the friction due to the bottom-wall: when $R e_{\text {fric }}$ is small, the bottom wall friction tends to impose the quasi-dimensionality of the flow. $l_{e d}$ is the eddy length scale associated to flow vortices. To take into account the multi-scale aspect of the flow, $\omega_{r m s}$ and its standard deviation are estimated over velocity fields re-normalized inside windows of $l_{e d}$ size for $l_{e d} \geqslant 14$ pixels (14 pixels correspond to the original data). Figure 11( $a$ ) illustrates case $l_{e d}=448$ pixels. The whole range of $l_{e d}$ is used to check the quasi-dimensionality.

Figure 7 shows that all the flows considered in our experiments are quasi-twodimensional. Direct numerical simulations of the two-dimensional Navier-Stokes equation electromagnetically forced as in this experiment and with a Rayleigh friction term to simulate the effect of bottom friction (see Rossi et al. 2005) generate results very close to figure 9 and to the energy spectrum of figure 15 . This provides extra confidence on the quasi-two-dimensional nature of our flows.

\section{Multi-scale flow topology}

\subsection{Dye visualizations}

Figure 8 shows dye (fluorescein) visualizations of the multi-scale flow generated by our multi-scale EM forcing in the case where $I=0.3 \mathrm{~A}$. The positions of the large and medium-sized magnets are indicated in figure 8 by $\mathrm{N}$ and $\mathrm{S}$. The three scales associated with the EM forcing do clearly appear as scales of the flow. In fact, the flow is similar to the schematic fractal flow of figure 1 with stagnation points at different scales forming part of a cat's eyes within cat's eyes multi-scale topology. Figure $8(a)$ shows instances in the time evolution of the flow within the horizontal $80 \mathrm{~cm} \times 80 \mathrm{~cm}$ square area containing all the magnets and centred at the stagnation point between the two largest magnets. The initial spatial distribution of fluorescein when the forcing is switched on $(t=0)$ is random; after 30 s (i.e. $t u_{r m s} / L_{E}=1$ where the Eulerian integral length-scale $L_{E}$ is defined in $\S 4.3$; PIV-obtained values of $L_{E}$ and $u_{r m s}$ are given in table 1), the dye visualization shows some clear closed loops at the smallest scales of forcing (M10) while the larger-scale loops are not yet closed, but already strongly correlated to the positions of larger-scale magnet pairs. There are different time scales of the flow associated with the different length scales, and the smallest magnets are those that are the fastest in generating closed loops. By $t=80 \mathrm{~s}$, the cat's eyes within cat's eyes multi-scale flow topology is evident over three fractal iterations and remains steady in time as a result of the forcing itself being steady in time.

Figure $8(b)$ shows a different realization of the bottom left-hand quarter of the same flow (the largest M160 magnets are on the right-hand side of the picture) after $75 \mathrm{~s}$ from start of forcing (i.e. $t u_{r m s} / L_{E}=2.5$ ). Three different colours are used in this visualization so as to show the intermediate and small-scale structure of the flow. 

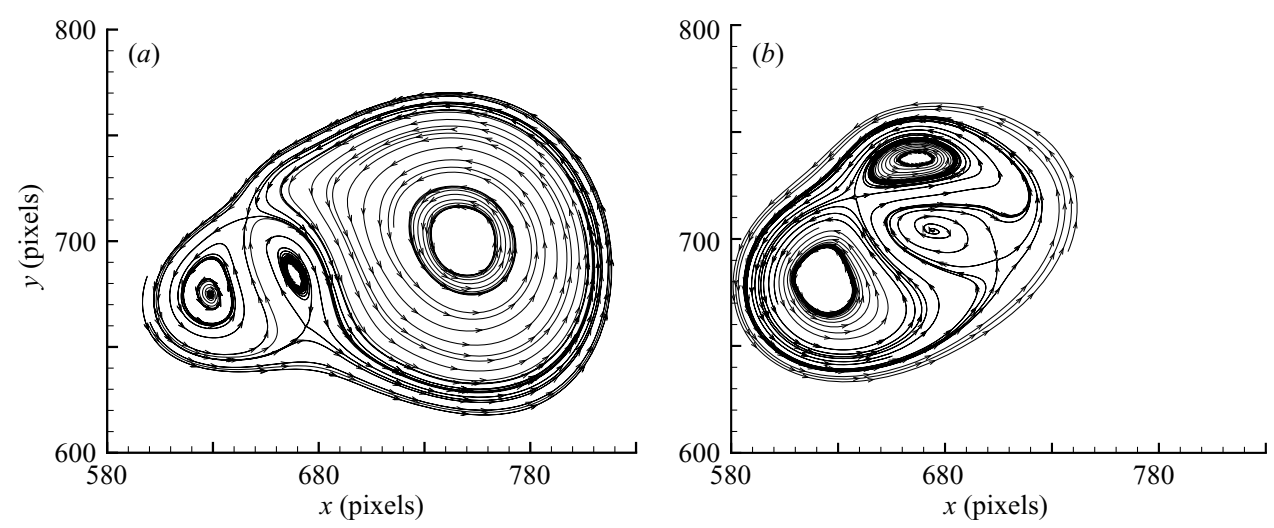

FIgURE 10. PIV measurements, streamlines at small scales for $I=0.3 \mathrm{~A}$ and $I=1 \mathrm{~A}$ (frame $80 \mathrm{~cm}$ ).

Some characteristic length scales are also given on the picture. It is clear that scales of EM forcing correspond to generated flow scales and to controlled stagnation points. Figure $8(b)$ also clearly shows the straining effect that some stagnation points have on the dye at various scales (see for example the orange tilted V shapes above visibly hyperbolic stagnation points relating to the M40 and M10 magnets).

\subsection{PIV and multi-scale analysis}

In figure 9, we present PIV measurements, one of the entire flow (i.e. within the $80 \mathrm{~cm} \times 80 \mathrm{~cm}$ frame containing all the magnets, figure $9 a$ ) and the other of a quarter of the flow $(40 \mathrm{~cm} \times 40 \mathrm{~cm}$ frame corresponding to the lower right-hand quarter of the $80 \mathrm{~cm} \times 80 \mathrm{~cm}$ frame, figure $9 \mathrm{~b}$ ) generated by an electrical current of $I=0.3 \mathrm{~A}$ (as in the flow visualizations presented in $\S 4.1$ ). The PIV measurements are sufficiently well defined in spatial resolution to catch the fractal topology of the velocity field accurately. In fact, the flow velocity field topology (figure 9) is in clear agreement with that obtained by dye visualization (figure 8 ). The largest-scale stagnation point controlled by the two M160 magnets is well defined. The ' 8 in 8 ' flow topology is apparent in the velocity field, with its three iterations clearly linked to the fractal set of magnets. The maximum velocities appear above the magnets M160 and M40. The brine flows above magnet-pairs M40 in two different directions (north and south) with an asymmetry due to interference from the larger-scale flow forcing M160. Nevertheless, the forcing at scale M40 is strong enough to impose its own stagnation point.

In figure 10, we plot small-scale flow streamlines extracted from PIV measurements taken in the $80 \mathrm{~cm} \times 80 \mathrm{~cm}$ frame for $I=0.3 \mathrm{~A}$ and $I=1 \mathrm{~A}$. The topology exhibits two hyperbolic stagnation points associated with three eddies. It should be noticed that the small-scale flow going up in between the two smaller eddies when $I=0.3 \mathrm{~A}$ results from the forcing of the south M10 magnet. The agreement with the small-scale flow structure in the upper right-hand corner of figure $8(b)$ is striking. It is important to point out that the topology of this small-scale-flow remains unchanged over the entire range of currents (and therefore flow intensities) studied here (compare figures $10 a$ and $10 b$ ). Furthermore, the position of the hyperbolic stagnation point at (xpix $=640$ 650 , ypix $=710-720$ ) does not vary with $I$ as much as that of the other hyperbolic stagnation point in figure 10. It is therefore imposed by an M10 magnet pair, and it 
can be claimed that the flow topology at small scales is controlled by the small-scale forcing. The main change with increasing $I$ resides in the large eddy becoming smaller and moving closer to the hyperbolic stagnation point at xpix $=640-650$, ypix $=710$ 720). This change with increasing $I$ reflects the interference of the outer scales (M40, M160) on the inner ones. This interference increases as $I$ increases and the bottom friction is gradually overcome, i.e. as the ratio of the viscous time to the advection time over the extent of the flow structure considered increases. This ratio is given by $\left(H^{2} / v\right) /\left(L_{\text {struc }} / u_{\text {rms }}\right)$. The size of the flow structure in figure 10 is $L_{\text {struct }} \approx 200$ pixel which makes this ratio equal to 1.4 for $I=0.3 \mathrm{~A}$ and 16 for $I=1 \mathrm{~A}$. Hence, the change of streamline shape with increasing $I$ is related to inertial transfers between the scales. We report, however, that for $I<0.3 \mathrm{~A}$, the streamline shape is very similar to that for $I=0.3 \mathrm{~A}$ in figure 10 .

We stress the conclusion that varying the intensity of the electric current over the approximate two decades tried here allows us to change the intensity of the flow without changing its topology. Consequently, this topology is found to be stable over more than one order of velocity magnitude.

\subsection{Spatial correlations}

Given the PIV velocity field, the two-dimensional spatial correlation function, $R_{2 D}$, is calculated by averaging over all points (denoted $\boldsymbol{x}$ ), i.e.

$$
R_{2 D}(\boldsymbol{r})=\langle\boldsymbol{u}(\boldsymbol{x}) \cdot \boldsymbol{u}(\boldsymbol{x}+\boldsymbol{r})\rangle
$$

Both coordinates of $\boldsymbol{r}$ are chosen between $-0.89 L_{P I V}$ and $0.89 L_{P I V}$ so as to avoid statistical problems caused by the edges of the PIV field. Denoting by $\Delta_{r}$ the spatial grid size of the PIV-measured velocity field, the one-dimensional spatial correlation, $R_{1 D}(r)$, is the angular average of $R_{2 D}$. In practice, we average over all vectors $r$ such that $r-\Delta_{r} / 2 \leqslant|\boldsymbol{r}|<r+\Delta_{r} / 2$.

The Eulerian integral length-scale $L_{E}$ is defined by

$$
L_{E}=\frac{1}{u_{r m s}^{2}} \int_{0}^{\infty} R_{1 D}(l) \mathrm{d} l .
$$

In table 1, we give the values of $L_{E}$ for different forcing cases. It can be seen that these values do not change significantly with increasing intensity of forcing, i.e. increasing Reynolds number. This observation suggests that the integral length scale $L_{E}$ of our multi-scale flow is controlled by the multi-scale spatial distribution of the forcing.

\subsection{Multi-scale analysis}

To illustrate and analyse the multi-scale topology of the flow, we apply to it averaging filters of various sizes. Specifically, the velocity field is averaged over a square window of size $L_{w}$ and a local average velocity is thus calculated. Examples of such filtered fields are given in figure 11. This filtering operation conducted at different length scales $L_{w}$ reveals very clearly the three iterations of the fractal (multiple-scale) flow generated by the EM forcing. Figure 11(a) shows only the large scale flow. In figure 11(b), the medium scale of the flow is apparent too. Finally, in figure 11(c), all the scales of the flow are present. Figure $11(d)$ is a zoom of figure 11(b) and figure 11(e) is a zoom of figure $11(c)$.

The energy spectrum of a flow is used to give an indication of the energy content of various sized eddies. In the present experiments, we can complement the energy spectrum which is obtained by Fourier transforming $R_{2 D}(\boldsymbol{r})$, with the flow patterns of different eddies of different sizes. In figure 12(a), we plot the filtered velocity 

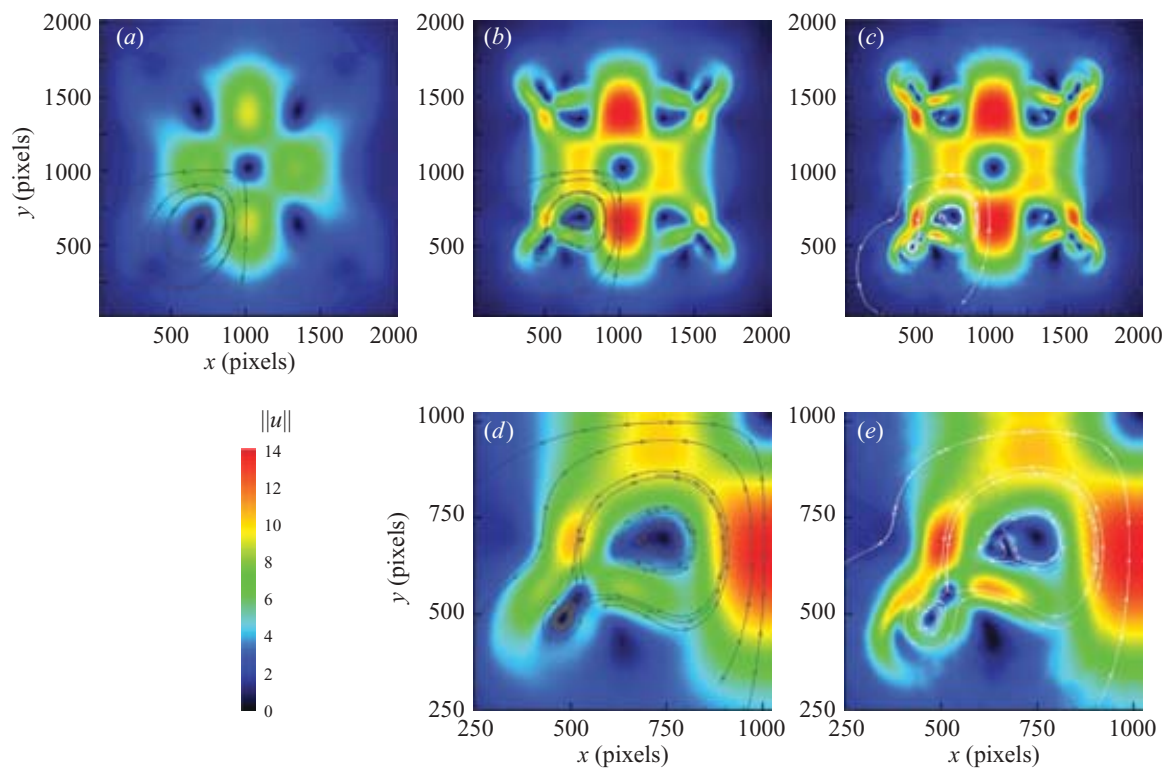

FigURE 11. Filtered PIV measurements by averaging in windows of size $L_{w}^{2}$. (a) $L_{W}$ about $178 \mathrm{~mm},(b, d) L_{W}$ about $44.5 \mathrm{~mm},(c, e) L_{W}$ about $8.3 \mathrm{~mm} ;(d)$ is a zoom of $(b),(e)$ is a zoom of $(c),\|u\|$ is the velocity intensity in $\mathrm{mm} \mathrm{s}^{-1}, 1$ pixel $\sim 0.397 \mathrm{~mm}$. Some streamlines are given by grey and white lines.
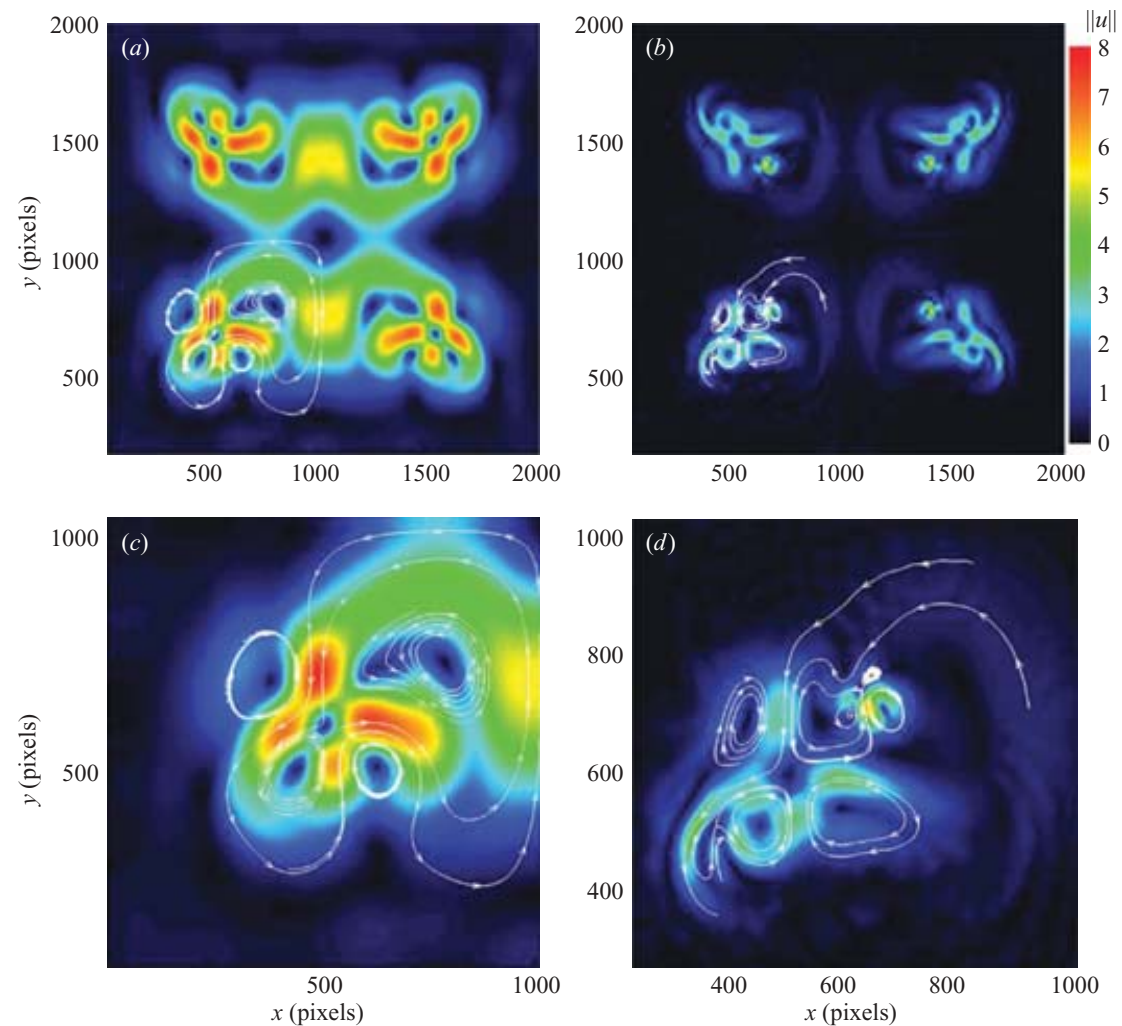

FIGURE 12. Flow for a range of scales obtained by the difference of the filtered flows of figure 11, $(a)$ figures 11(b)-11(a), $(b)$ figures 11(c)-11(b), $(c)$ zoom of $(a),(d)$ zoom of $(b)$. $\|\boldsymbol{u}\|$ is the velocity intensity in $\mathrm{mm} \mathrm{s}^{-1}$. 


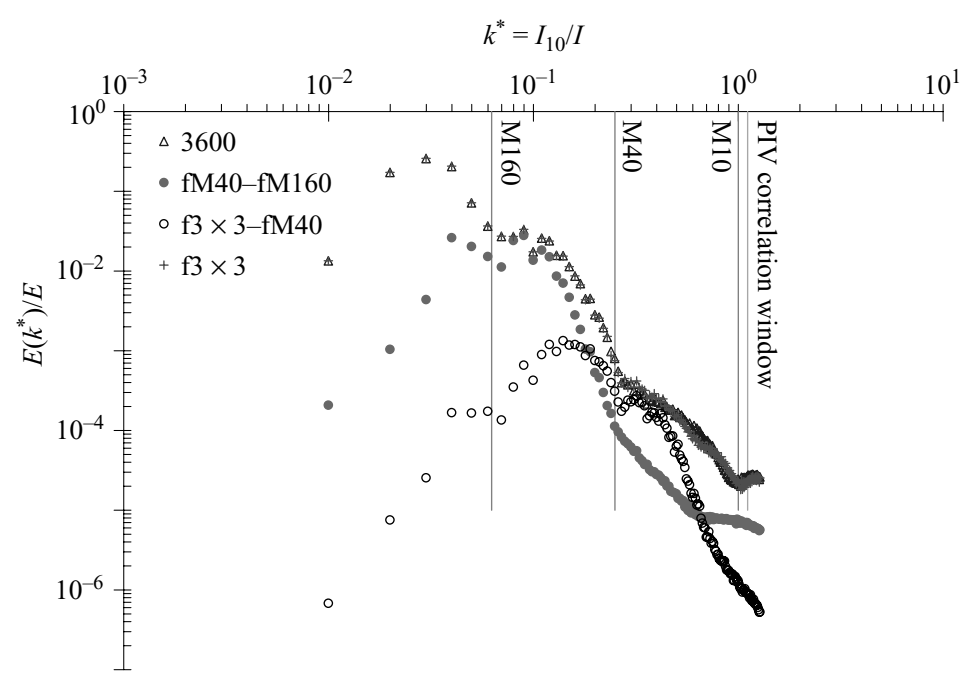

FIGURE 13. Energy spectra associated to multi-scale analysis, $3600\left(\operatorname{Re}_{2 D}\right)$ corresponds to the real flow; $\mathrm{f} 3 \times 3$ corresponds to the flow given in figures $11(c)$ and $11(e)$; fM40-fM160 corresponds to figures $12(a)$ and $12(c)$; fM40-fM160 corresponds to figures $12(b)$ and $12(d)$.

field of figure $11(b)$ from which we have subtracted the filtered velocity field of figure 11(a). Hence, figure 12(a) may be interpreted as the flow pattern of eddies of size between $178 \mathrm{~mm}$ and $44.5 \mathrm{~mm}$, thus corresponding to the part of the entire flow's energy spectrum determined by the magnets M160 and M40 (see figure 13). Similarly, figure $12(b)$ is a plot of the filtered velocity field of figure 11(c) from which we have subtracted the filtered velocity field of figure 11(b) and the resulting flow pattern corresponds to the part of the energy spectrum determined by magnets M40 and M10 (see figure 13). Figure 13 also provides comparisons between the energy spectrum of the entire unfiltered flow, the energy spectrum of the flow filtered at a scale $L_{w}$ smaller than $l_{2}$ (the length scale of M10) (the two spectra coincide), and the energy spectra of the flows in figures $11(a)$ and $11(b)$. It is clear that these latter two spectra are dominant contributions to the entire flow's spectrum at the scales where they are significant.

\section{Flow energy}

In this section, we study the flow's energy and its distribution in Fourier space. One of the non-trivial questions addressed here is whether the energy spectrum is continuous and power-law shaped, and whether the exponent $p$ of this power law is related to the fractal scaling of the flow's stagnation points by (1.1).

At $t=0$, the electrical current is suddenly switched on from zero to a constant value. This generates a flow which increases in energy over a short transient time until it soon reaches a constant energy value. Similarly to Paret et al. (1997), this transient is found to be fitted well by:

$$
u_{r m s}^{2}(t)=u_{r m s}^{2}\left(1-\exp \left(-a t v / H^{2}\right)\right)
$$

where $a$ is a positive dimensionless constant (examples of values of $a$, all close to 1 , are: $a=1.07$ for $I=0.04 \mathrm{~A}, a=1.34$ for $I=0.06 \mathrm{~A}$ and $a=1.46$ for $I=0.1 \mathrm{~A}$ ) and $u_{r m s}^{2}$ is the asymptotic value of $u_{r m s}^{2}(t)$. The short duration of the transient is therefore 


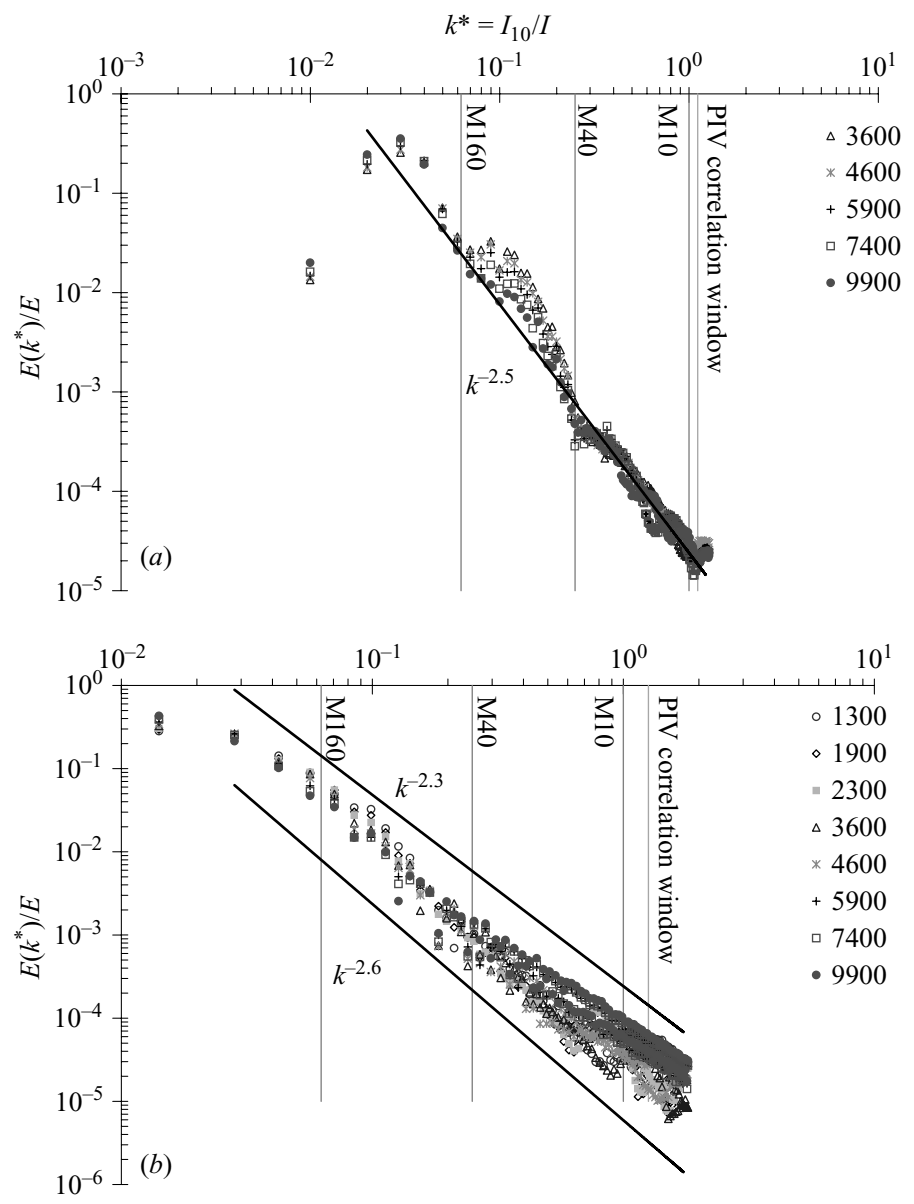

FIGURE 14. Flow energy spectrum for different Reynolds number $R e_{2 D}$. The three sizes of forcing (M10, M40, M160) as well as the PIV's correlation window size are indicated by vertical straight lines. (a) PIV frame $80 \mathrm{~cm}$. Diagonal straight line illustrates $k^{-2.5},(b)$ PIV frame $40 \mathrm{~cm}$. Diagonal straight lines illustrate $k^{-2.3}$ and $k^{-2.6}$.

about $(4 / a)\left(H^{2} / \nu\right)$; beyond this time the flow is established at its full energy and can be assumed to be quasi-stationary. The flow's quasi-stationarity is confirmed by the Eulerian velocity autocorrelation in time, $\langle\boldsymbol{u}(\boldsymbol{x}, t) \cdot \boldsymbol{u}(\boldsymbol{x}, t+\tau)\rangle$ (where the average is taken over $\boldsymbol{x})$ which we find to be very close to $u_{r m s}^{2}$ for $t$ larger than $(10 / a)\left(H^{2} / v\right)$. As an example which is typical of the general rule, when $I=0.1$ A and $\tau \simeq L_{E} / u_{r m s}$, then $\langle\boldsymbol{u}(\boldsymbol{x}, t) \cdot \boldsymbol{u}(\boldsymbol{x}, t+\tau)\rangle=0.996 u_{r m s}^{2}$.

\subsection{Energy spectra}

The energy spectrum of the flow is computed for the quasi-stationary state, and in figure 14 we plot it for a range of flow intensities (i.e. Reynolds numbers, $R e_{2 D}$ ).

The energy spectrum displays oscillations with a log-periodicity similar to the log-periodicity in size of the magnets (i.e. the forcing). These oscillations weaken significantly as the Reynolds number increases. We attribute this weakening to the increase of inertial interferences between scales which we identified in $\S 4.2$. Whilst the flow topology is unchanged when increasing $I$, the streamline eddy sizes seen, for example, in figure 10 tend to become more uniform. This streamline change 


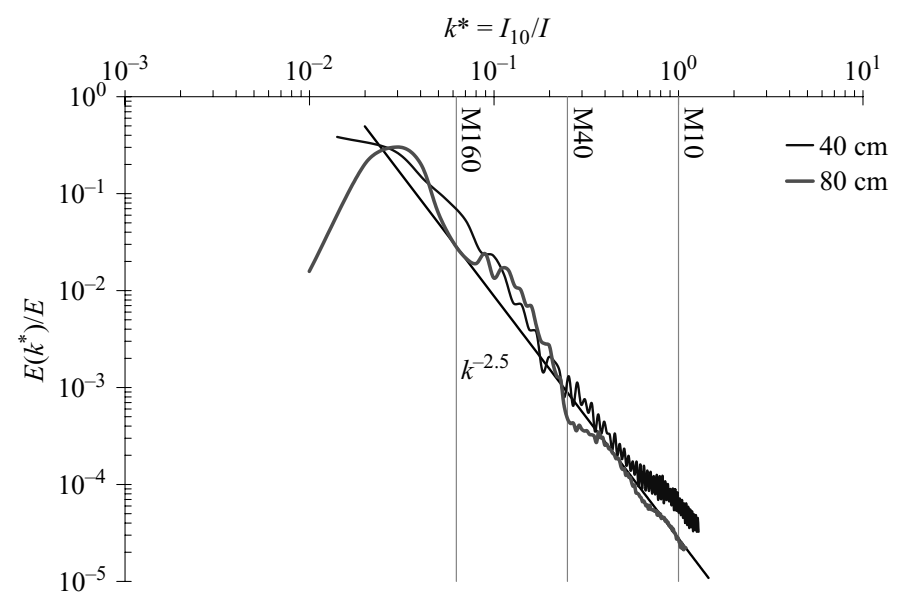

FIgURE 15. Average flow energy spectrum for frame $80 \mathrm{~cm}$ and $40 \mathrm{~cm}$. The three sizes of forcing (M10, M40, M160) are indicated by vertical straight lines. The diagonal straight line gives $k^{-2.5}$.

with increasing $I$ (i.e. increasing Reynolds number) is correlated with the increased smoothing which leads to a reasonably well-defined power-law shape of the energy spectrum, see figure $14(a)$ and the trend from $R e_{2 D}=3600$ to $R e_{2 D}=9900$.

Estimations of the exponent $p$ of this power-law energy spectrum vary between 2.3 and 2.6 for different flow intensities. However, as the Reynolds number increases, the energy spectrum becomes progressively closer to a power-law shape with $p=2.5$, see figure $14(a)$.

The flow energy spectra are very similar for different Reynolds numbers. In particular, the outer and inner length scales $L$ and $\eta$ over which $E(k)$ extends as a more or less well-defined power law are determined by the multi-scale range of the EM forcing and are the same at all Reynolds numbers. We therefore have separate control over $(L / \eta)$ and Reynolds number in the present class of flows.

We take advantage of the similarity of the energy spectra at different Reynolds numbers and calculate two representative energy spectra, one in PIV frame $80 \mathrm{~cm}$ and the other in PIV frame $40 \mathrm{~cm}$, by averaging energy spectra over different Reynolds numbers. Figure 15 shows these two average energy spectra. There is a good agreement with a power-law energy spectrum of exponent $p$ close to 2.5 in both PIV frames.

Note that the spectral exponent $p$ of our multi-scale flows differs from, and in fact lies between, the values 5/3 (e.g. Kraichnan 1967; Paret \& Tabeling 1997; Julien et al. 1999) and 3 or higher (Boffeta et al. 2005) which arise when statistically stationary two-dimensional turbulence is forced only at the small scales or only at the large scales, respectively. Clercx \& Van Heijst (2000) also obtain exponents $p$ between $5 / 3$ and 3 in their numerical simulations of decaying high-Reynolds-number twodimensional turbulence with no-slip boundary conditions and argue that their spectra and values of $p$ reflect the influence of these boundary conditions. However, our flows are different from theirs in that ours are non-decaying and effectively laminar. Also, the energy spectra of their turbulent flows are strongly Reynolds-number dependent whereas ours are not. More importantly, perhaps, the size of our tank is very large compared to our largest area of PIV measurements $(80 \mathrm{~cm}$ PIV frame which covers much more than the area of direct action of the magnets) and the flow outside this area is very weak. In fact, fluid velocities are not significant near the boundaries of 

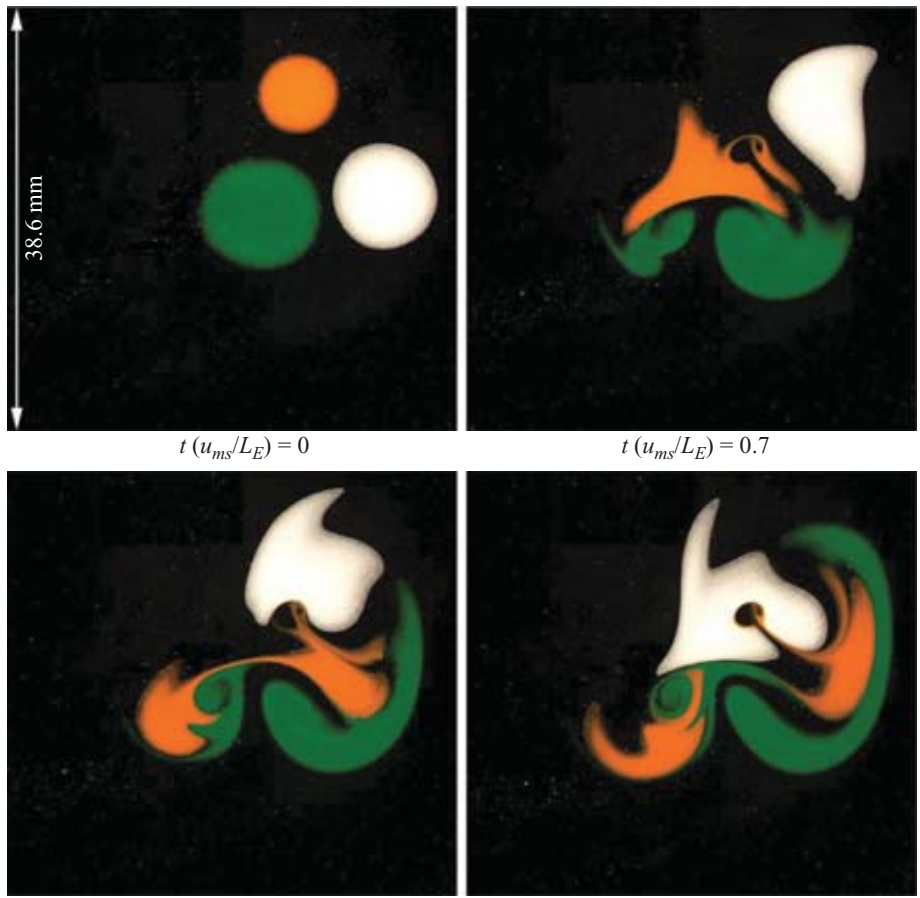

$t\left(u_{m s} / L_{E}\right)=1$

$t\left(u_{m s} / L_{E}\right)=1.4$
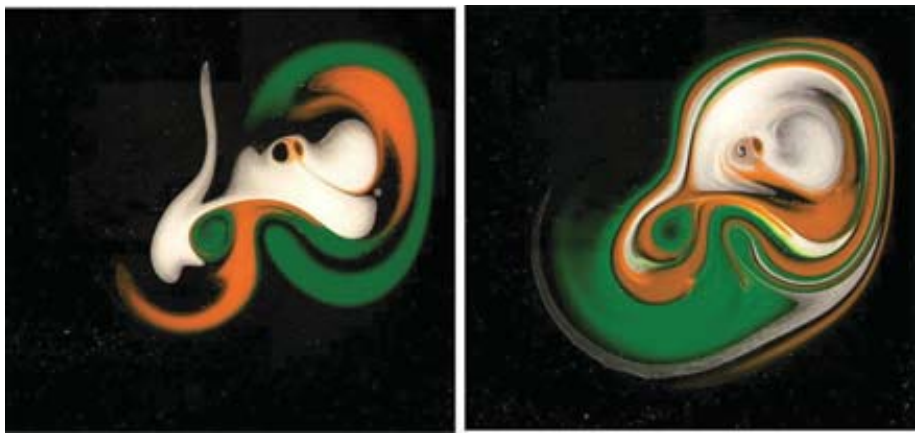

$t\left(u_{m s} / L_{E}\right)=1.7$

$t\left(u_{m s} / L_{E}\right)=4.8$
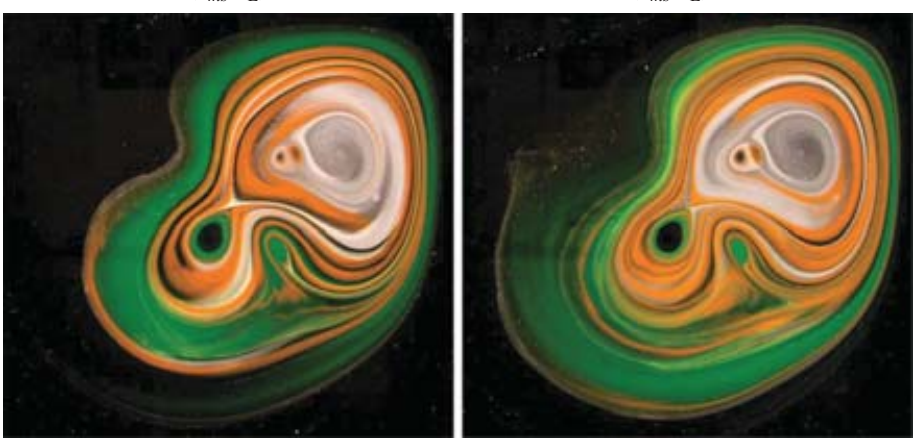

$t\left(u_{m s} / L_{E}\right)=9.5$

$t\left(u_{m s} / L_{E}\right)=14.1$

FIGURE 16. Quarter of the flow illustration of dye mixing and stretching with a multi-scale electromagnetic forcing constant in time. At $t<0$, three blobs of dye (orange, green and white) are placed above the free surface. At $t=0$, the forcing is switched $\mathrm{ON}$ to $I=0.3 \mathrm{~A}$. The south big magnet (M160) is on the right-hand side of the pictures and the top right-hand corner is close to the large scale stagnation point, see figure 9. The physical length scale is indicated on the first picture. 
the tank. We can therefore argue that the energy spectra of our non-decaying flows are not affected by the boundaries. In fact, our flows are clearly and qualitatively different from those of Clercx \& Van Heijst (2000) and we conclude from the results and discussion in this section and $\S 4.4$ that the unusual spectral signatures that we obtain (figure 15 and $p=2.5$ ) reflect the multi-scale topology of our flows and result from the unusual multi-scale property of our forcing.

\subsection{Fractal dimension and control of the energy spectrum}

The fractal dimension $D_{s}$ of the hyperbolic stagnation points that we force into the flow, which is the same as the fractal dimension of the centres of mass of our magnet pairs, is given by $N_{s}=2^{n+3}-3$ (the powers of 2 result from $R=4$, and the coefficient -3 accounts for the single stagnation point at the largest scale, see $\S 2.1$ ) and $N_{s} \sim\left(l_{0} / l_{n}\right)^{D_{s}}$ where $N_{s}$ is the number of all stagnation points in our flow down to length scale $l_{n}$ (as opposed to the number density $n_{s}$ defined in the text before equations (1.1) and (1.2)). As a result, $D_{s}=0.5$. To be precise, this is the fractal dimension of the forced stagnation points, but there are also some other stagnation points in the flow which are not directly forced but result from the forming of the flow. It is not possible at this stage to meaningfully fit $N_{s}\left(l_{0} / l_{n}\right)$ as a power-law function of $l_{0} / l_{n}$ and obtain $D_{s}$ because we do not have a large enough number of stagnation points in our flows. Hence, we assume that $D_{s}$ represents the fractal dimension of the set of all stagnation points and leave this issue for future study using numerical simulations of our fractal-like flows (see Rossi et al. 2005) where $L / \eta$ can be made larger than it can in the laboratory. (See the Appendix for further details on $D_{s}$.)

Our values $p=2.5$ and $D_{s}=0.5$ sum up to give $p+D_{s}=3$ which is relation (1.1). This agreement is striking and suggests that the energy spectrum of the flow might indeed be controllable by multi-scale forcing of the distribution of stagnation points as well as of the scales of energy input. However, there is a need to check the validity and limitations of $p+D_{s}=3$ with other forcing geometries (i.e. other values of $D_{s}$ ) and with time-dependent forcing which are beyond the capabilities for the present paper. Note, finally, that the exponents $p$ of the power-law energy spectra of our class of flows are effectively found to be in the range between 2.3 and 2.5 , and that detailed considerations in the Appendix about the fractal dimension $D_{s}$ lead to values of $D_{s}$ between 0.7 and 0.5 .

\section{Stirring and Lagrangian statistics}

Following the structure of our introduction in $\S 1$ and having obtained evidence in support of the idea that the multi-scale EM forcing controls the spatial distribution of stagnation points which, in turn, controls the power-law shape of the energy spectrum, we now investigate the extent in which these stagnation points also control the stirring and, in particular, the Lagrangian statistics of pair dispersion. This section is therefore devoted to the study of the Lagrangian statistics of our laminar multi-scale flows.

\subsection{Illustration of stirring}

Figure 16 provides an illustration of the stirring and stretching of three initial blobs of different colours: orange, green and white. An additional snapshot $\left(t u_{r m s} / L_{E}=2.5\right)$ from the same time-series is given in figure $8(b)$. This time-series clearly shows a stretching mechanism which generates a well-defined field of alternating colours and long interfaces. Initial positions of blobs matter: whereas the orange and green blobs are directly affected by the small scales, the white blob is only affected by the small scales after one turnover time. 
At short times, $t u_{r m s} / L_{E} \lesssim 1$, the stirring and stretching appears to be dominated by the two small scales (M10 and M40). For longer times, $t u_{r m s} / L_{E}>1$, the trajectories eventually close up and the large scales make a more important contribution to the process of stirring and mixing. Interpreting $\left(k^{3} E(k)\right)^{-1 / 2}$ as being an average eddy turnover time at length scale $2 \pi / k$, then these observations agree with the fact that the eddy turnover time is smaller for smaller length scales as a consequence of $E(k) \sim k^{-2.5}$.

\subsection{Computations from experimental data}

Lagrangian statistics are computed from time resolved PIV measurements by integrating trajectories of fluid elements. To remove the noise at small scales, a $3 \times 3$ point averaging process is used taking advantage of the $56 \%$ correlation windows overlap. Even though most of the flow is within the PIV $80 \mathrm{~cm}$ frame used here, a few fluid elements move in and out of that measurement area. In order to keep the total number of fluid elements constant in our pair and single fluid element statistics, the flow has been artificially closed at the size of the tank in accordance with the continuity equation so as to ensure mass conservation. Except for ensuring the quality of our Lagrangian statistics, this artificial procedure does not affect them because:

(i) only fluid elements in the measurement area or crossing it are counted in the statistics;

(ii) the velocity of the flow outside the measurement area is more than 10 times smaller than $u_{r m s}$;

(iii) the turnover time scale in that outer area is extremely large (100 times) compared to the turnover time $L_{E} / u_{r m s}$ which is itself larger than the Lagrangian correlation time $T_{L}$;

(iv) the duration of the PIV data acquisition and Lagrangian tracking is, by design, ten times smaller than the turn-over time in the outer area so as to guarantee that fluid elements do not loop at the size of the tank.

The calculated accuracy of the numerical scheme which we used to compute trajectories gives a potential error of about $10^{-3}$ pixel per time step (data checked). This is much smaller than the measurement noise $(2 \%)$ which is about 0.022 pixel per time step. Since the numerical accuracy is more than 20 times greater than the displacement on trajectories caused by noise, the numerical accuracy is sufficient for our purposes. The time step for our Lagrangian integrations is 11 times smaller than the time step for the PIV measurements.

In the remainder of this paper, we calculate Lagrangian statistics obtained from the PIV velocity field corresponding to $I=0.1 \mathrm{~A}$ and $R e_{2 D}=1300$. There are two reasons for this choice. (i) Time resolution is limited by memory and hard drive speed when continuous long time acquisition is needed, as is the case here, and our best time-resolved data were obtained for $I \leqslant 0.1$ A. (ii) At values of $I$ significantly smaller than $0.1 \mathrm{~A}$, the bottom friction makes the flow so slow away from the magnets that it is not captured with enough accuracy by our PIV in a portion of the flow which increases with decreasing $I$.

\subsection{Fluid element trajectories and Lagrangian time}

\subsubsection{Fluid element trajectories}

In figure 17(a) we plot various fluid element trajectories. We integrate many groups of three trajectories initialized at many randomly positioned equilateral triangles of side length equal to 1 pixel. The strong dispersion of fluid elements when they encounter a hyperbolic stagnation point appears clearly at every scale of the flow. Of 


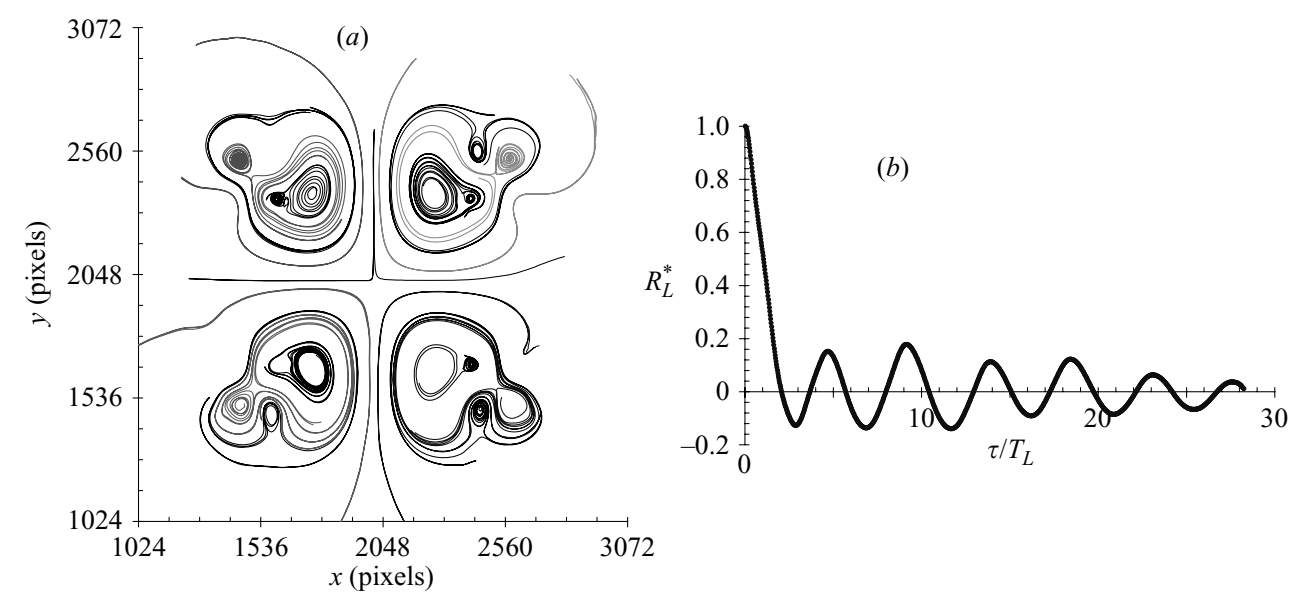

Figure 17. (a) Selection of fluid elements trajectories, at $t=0$ the forcing is switched $\mathrm{ON}$; 1 pixel $\sim 0.397 \mathrm{~mm} ;(b)$ dimensionless Lagrangian correlation, $R_{L}^{*}$, versus dimensionless time, $\left(\tau / T_{L}\right) R_{L}(\tau)=\left(1 / N_{t}\right) \sum_{t}\langle\boldsymbol{u}(t) \cdot \boldsymbol{u}(t+\tau)\rangle$.

course, this might be expected in our flow, at least after the initial transient when it settles into a quasi-stationary state and trajectories follow streamlines.

In addition, even if the flow is quasi-two-dimensional, the flow measurements are not perfectly two-dimensional (sensu stricto) as can be seen from some particle trajectories and figure 6.

\subsubsection{Lagrangian autocorrelation function and time scale}

The Lagrangian autocorrelation function $R_{L}(\tau)=\overline{\boldsymbol{u}_{L}(t) \cdot \boldsymbol{u}_{L}(t+\tau)}$ calculated by averaging over time $t$ and over many trajectories $(\mathrm{d} / \mathrm{d} t) \boldsymbol{x}\left(t, \boldsymbol{x}_{0}\right)=\boldsymbol{u}_{L}\left(t, \boldsymbol{x}_{0}\right)$ starting at random initial positions $\boldsymbol{x}_{0}$, is given in figure $17(b)$. The oscillations around zero are the consequence of the symmetry of the frozen flow and its periodicities at the three scales of forcing. Note that the intensity of these oscillations is decreasing for large $t / T_{L}$.

The Lagrangian correlation time $T_{L}=\int_{0}^{\infty} R_{L}(\tau) / R_{L}(0) \mathrm{d} \tau$ is $T_{L} \simeq 28.15 \mathrm{~s} \simeq$ $0.37 L_{E} / u_{r m s}$.

\subsection{Fluid element dispersion}

In this section, the Lagrangian trajectories $(\mathrm{d} / \mathrm{d} t) \boldsymbol{x}\left(t, \boldsymbol{x}_{0}\right)=\boldsymbol{u}_{L}\left(t, \boldsymbol{x}_{0}\right)$ and their statistics are calculated starting from random initial positions $x_{0}$ at time $t=0$, which is the time when the forcing is switched on. These trajectories are integrated until $t=30 T_{L}$, and we extract pair statistics $\overline{\Delta^{2}}(t)$ and single fluid element statistics $\overline{\left(\boldsymbol{x}(t)-\boldsymbol{x}_{0}\right)^{2}}$, the averages being carried out over many pairs and many trajectories, respectively.

The results are plotted in figure 18. Pair statistics are initialized with initial separation $\Delta_{0}=1$ pixel which is smaller than all the length scales of the flow. Statistics such as mean square pair separations are sensitive to the choice of $\Delta_{0}$, but the turbulent diffusivity $(\mathrm{d} / \mathrm{d} t) \overline{\Delta^{2}}$ is much less sensitive, as shown by Nicolleau \& $\mathrm{Yu}$ (2004). In figures $18(a)$ and $18(b)$ we therefore plot both $\overline{\Delta^{2}}$ and its time-derivative (obtained by linear estimation) as functions of time. These two curves reveal two distinct approximate power laws $\overline{\Delta^{2}} \sim t^{\gamma}$, one with $\gamma \approx 3.1$ for times $t \leqslant T_{L}$, and one with $\gamma=2.3$ for longer times.

The exponent $\gamma \approx 3.1$ obtained for times shorter than $T_{L}$ is close to the power 3 of Richardson's law for isotropic homogeneous turbulence (Richardson 1926; Obukhov 

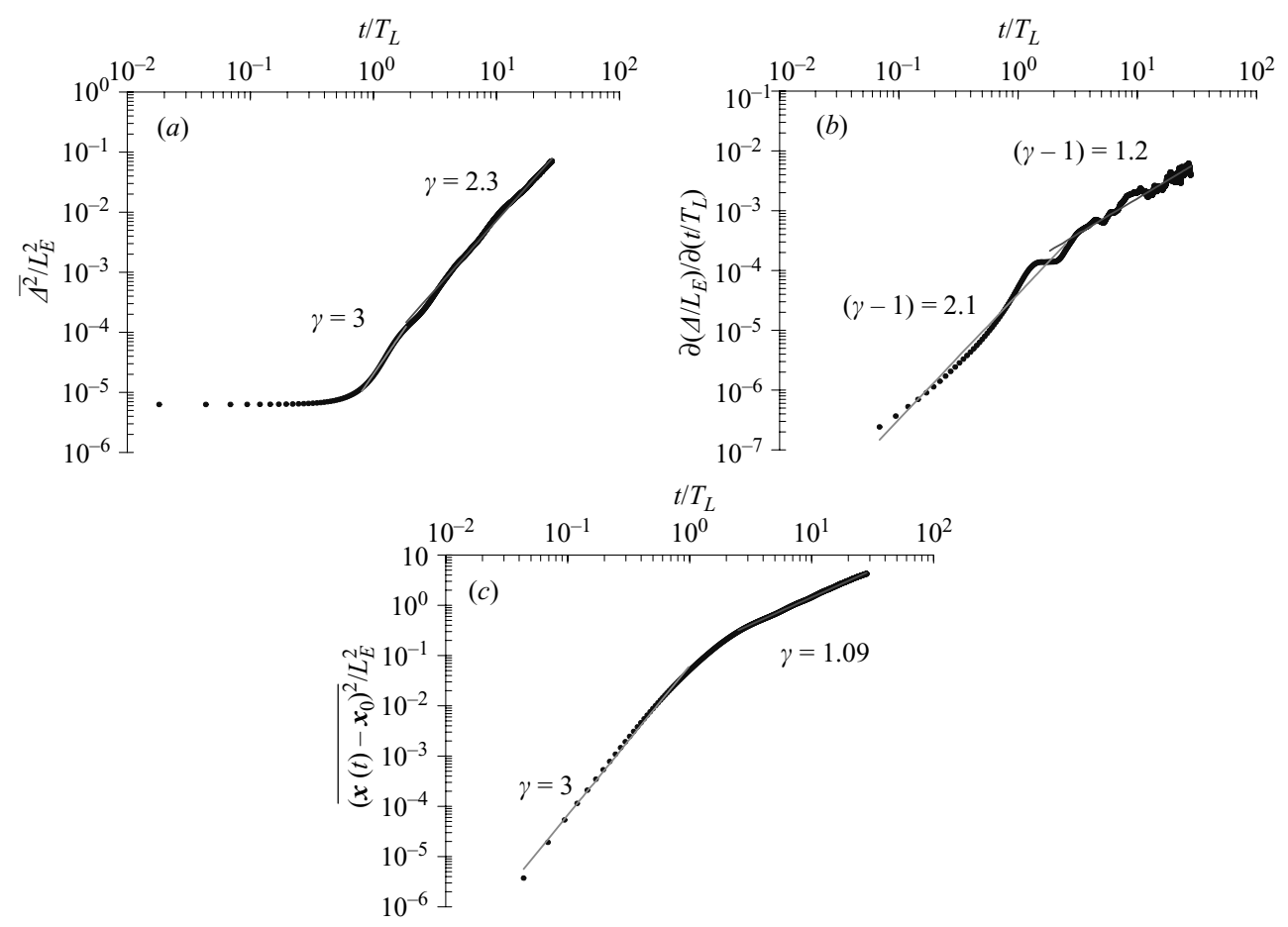

FIGURE 18. Fluid element dispersion versus $t / T_{L}:(a, b)$ two elements, $(c)$ one element. (a) $\left(\Delta / L_{E}\right)^{2} ;(b) \partial\left(\Delta / L_{E}\right)^{2} / \partial\left(t / T_{L}\right) ;(c) \overline{\left(\boldsymbol{x}(t)-\boldsymbol{x}_{0}\right)^{2}} / L_{E}^{2}$. At $t=0$ the forcing is switched ON. Each time's dot is a PIV measurement. (1 129161 pairs).

$1941)$ even though our multi-scale flow is not turbulent but laminar $\left(\operatorname{Re}_{3 D} \sim 10\right)$. The Richardson exponent $\gamma=3$ has been observed, or at least claimed to have been observed, in laboratory experiments (Julien et al. 1999; Ott \& Mann 2000) and numerical simulations (e.g. Boffeta \& Sokolov 2002; Ishihara \& Kaneda 2002; Goto \& Vassilicos 2004) of three-dimensional and two-dimensional (inverse cascading) isotropic turbulence. However, the approximate $\overline{\Delta^{2}} \sim t^{3}$ behaviour in a range of times $t$ bounded from above by $T_{L}$ in our flow is not a Richardson law because $\overline{\Delta^{2}}$ does not grow above $L_{E}^{2}$ and does not even grow proportionally to $t$ when $t$ exceeds $T_{L}$. In fact, $\overline{\Delta^{2}}$ remains smaller than $L_{E}^{2}$ for as long as we measure, i.e. for all times $t$ less than $30 T_{L}$, and perhaps longer. Furthermore, $\overline{\Delta^{2}} \sim t^{2.3}$ is a good approximation for times $t$ larger than $T_{L}$; at these long times, the exponent $\gamma$ is therefore not only larger than 1 , but even larger than 2, which would have been its value in a steady laminar shear flow.

The integral length scale $L_{E}$ is a bit more than 10 times smaller than the size of the tank. Hence, the second regime where $\gamma=2.3$ cannot be the result of a limiting length reached during dispersion (such as the size of the tank; in fact every single pair separation remains significantly smaller than half the tank size throughout our measurements) as might be the case of the second regime observed but not commented on by Julien et al. (1999). In fact, from observing and timing our multi-scale laminar flow, the turnover time associated with the smallest scales is slightly smaller than $T_{L}$, the turnover time associated with the medium scales is about $3 T_{L}$ and the turnover time of the large scales is approximately $8 T_{L}$. Hence, in the present context, $T_{L}$ should be thought of as an inner time scale rather than an outer one, the outer time scale being an order of magnitude larger, in fact about $8 T_{L}$ which is close to $L_{P I V} / 2 u_{r m s}$ 

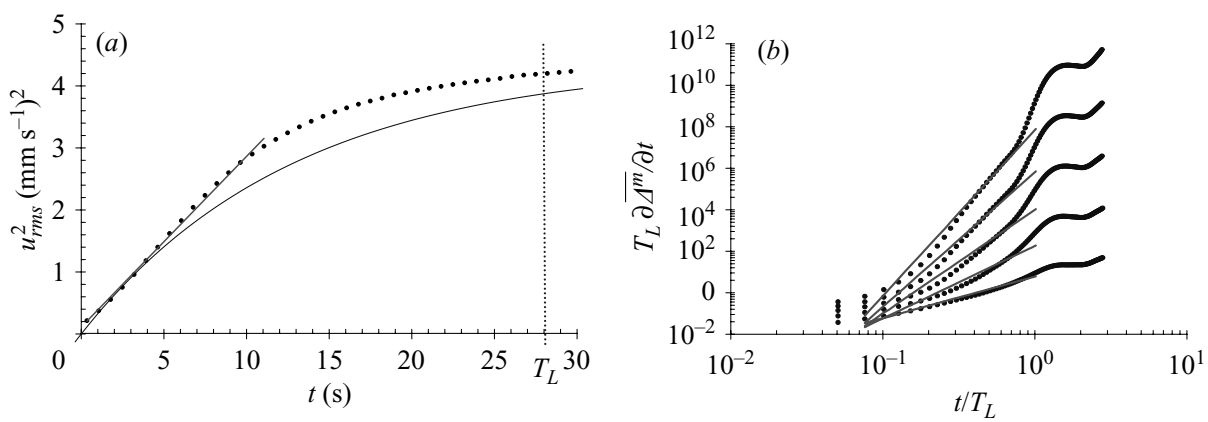

FIGURE 19. (a) Energy growth during transient. The lines correspond to $u_{r m s}^{2}=u_{\infty}^{2}(1-$ $\left.\exp \left(-a t v / H^{2}\right)\right)$ with $a=1.46$ and to the linear fit of $u_{r m s}^{2}(t)$ for short times; $(b)$ time derivative of $\overline{\Delta^{m}}$ during the energy transient. The straight lines show the ballistic dispersion associated to accelerated flow for each order, with $\overline{\Delta^{m}} \sim t^{3 m / 2}$ and $m=2,3,4,5,6$, (1 129161 pairs.)

here. It is in this range of time scales that $\overline{\Delta^{2}} \sim t^{2.3}$ is a reasonable approximation of the data. Before attempting to explain what causes each one of these two regimes, $\gamma=3$ and $\gamma=2.3$, in the following two subsections, we examine the single fluid element statistics of figure $18(c)$.

Our results in that figure are in agreement with Taylor's (1921) mathematical result that $\overline{\left(\boldsymbol{x}(t)-\boldsymbol{x}_{0}\right)^{2}} \sim t$ for $t \gg T_{L}$ (Taylor's (1921) result is valid if $T_{L}$ is finite, which is the case here). The oscillatory nature with significant amplitudes of the Lagrangian autocorrelation function over long times (figure 17b) means that long-range correlations exist which must affect pair statistics but which introduce cancellations in the definition of the Lagrangian correlation time $T_{L}$ with the result that $T_{L}$ sums out to be too small to represent these long-range correlations. As a result, the longrange behaviour $\overline{\left(x(t)-x_{0}\right)^{2}} \sim t$ coexists with the non-trivial intermediate range pair dispersion behaviour $\overline{\Delta^{2}} \sim t^{2.3}$, the intermediate range being over a decade of time scales starting at about $T_{L}$. The power $\gamma$ being significantly larger than 1 indicates clearly that pairs remain correlated in this intermediate range of times.

Finally, figure 18(c) provides an important clue for understanding the range $t \leqslant T_{L}$. In that range, $\overline{\left(\boldsymbol{x}(t)-\boldsymbol{x}_{0}\right)^{2}} \sim t^{3}$ rather than the short-time ballistic expectation $t^{2}$. This is explained in the following subsection.

\subsection{The energy transient}

Figure 19(a) reveals the linear increase with time of the total kinetic energy of the flow during the initial transient: $u_{r m s}^{2}(t) \sim t$ for $0<t \lesssim T_{L} / 2$. This energy growth can also be fitted by $u_{r m s}^{2}(t)=u_{r m s}^{2}\left(1-\exp \left(-a t v / H^{2}\right)\right)$ as mentioned in $\S 5$, which agrees with the linear time growth for $0<t \lesssim(1 / a)\left(H^{2} / v\right)$. As it happens, in the present case $(1 / a)\left(H^{2} / \nu\right) \approx T_{L} / 2$.

The consequence is that the dispersion's ballistic regime during transient is not a $t^{2}$ law but a $t^{3}$ law because of the linear increase of the kinetic energy, $u_{r m s}^{2}(t) \sim t$ at times shorter than about $T_{L}$. Specifically, $\overline{\left(\boldsymbol{x}(t)-\boldsymbol{x}_{0}\right)^{2}} \sim t^{3}$ and $\overline{\Delta^{2}} \sim t^{3}$, i.e. $\gamma=3$, as a direct result of ballistic dispersion during transient. This transient ballistic regime is confirmed by the approximate result that $\overline{\Delta^{m}} \sim t^{3 m / 2}$ for times smaller than $T_{L}$ which we plot in figure $19(b)$ for $m=2,3,4,5,6$.

The transient sets in with the sudden forcing step which causes relatively short-lived near-constant accelerations to exist uniformly in the flow until competing EM and bottom-friction forces balance and a quasi-stationary flow state is established. 

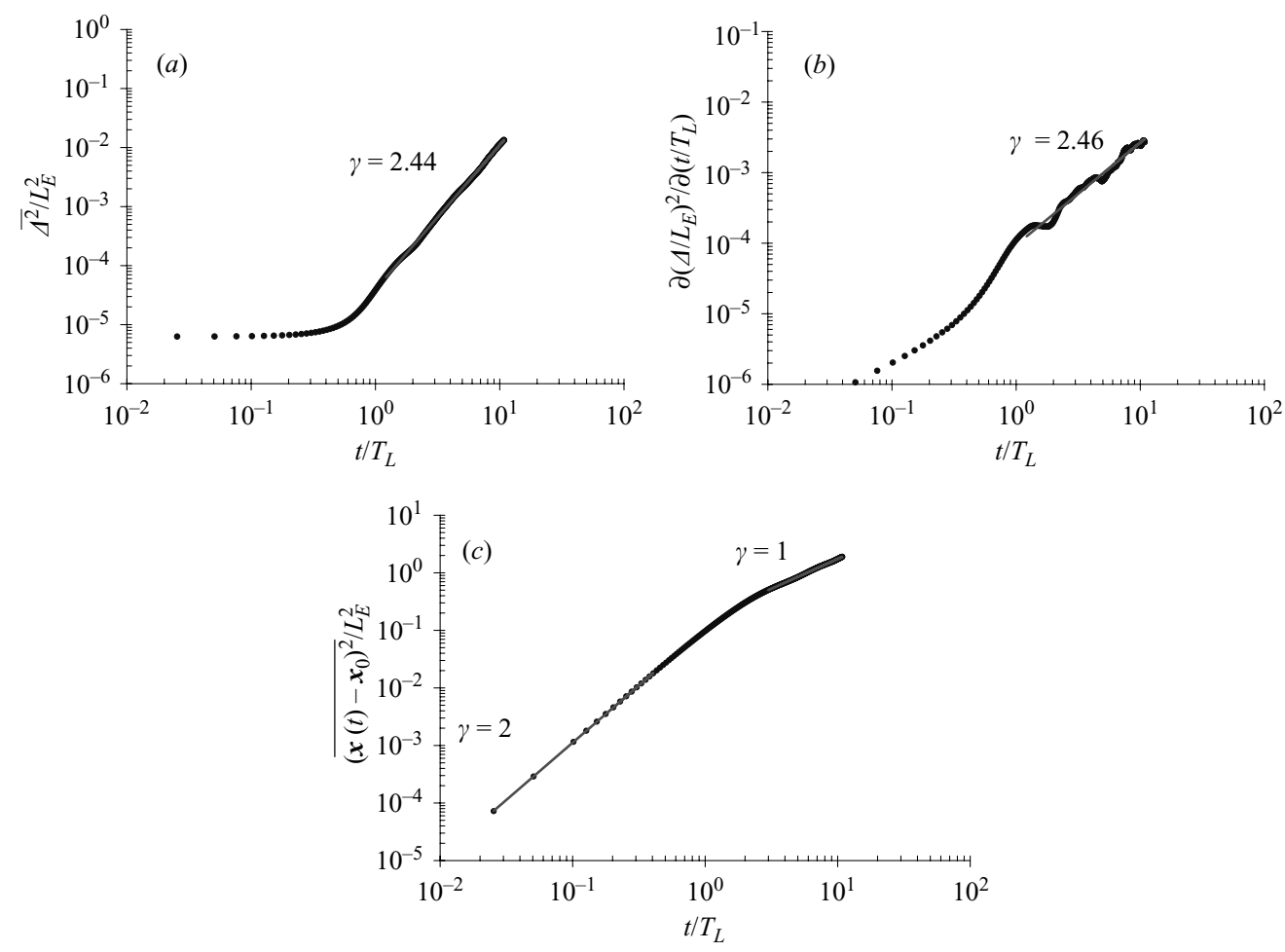

FiguRE 20. Fluid element dispersion for statistics started after energy transient $(t=0$, $\Delta_{0}=1$ pixel $)$ versus $t / T_{L}:(a, b)$ two elements, one element. $(a)\left(\Delta / L_{E}\right)^{2} ;(b) \partial\left(\Delta / L_{E}\right)^{2} / \partial\left(t / T_{L}\right)$; (c) $\overline{\left(\boldsymbol{x}(t)-\boldsymbol{x}_{0}\right)^{2}} / L_{E}^{2} \cdot(1619484$ pairs.)

\subsection{Quasi-stationary state}

To understand better the dispersion in the intermediate time range which follows the initial transient, we integrate fluid element trajectories initialized at random positions $\boldsymbol{x}_{0}$ at a time $t_{0}>(13 / a)\left(H^{2} / v\right)$. This way, Lagrangian trajectories are not contaminated by the energy transient as they are integrated in what is a quasistationary flow throughout. The Lagrangian autocorrelation function of figure $17(b)$ remains essentially unaffected and $T_{L}$ is only very slightly larger than the value $28.15 \mathrm{~s}$ obtained from figure $17(b)$.

\subsubsection{Single fluid element and pair dispersion}

We obtain a conventional initial ballistic regime $\overline{\left(\boldsymbol{x}(t)-\boldsymbol{x}_{0}\right)^{2}} \sim t^{2}$ for $t<T_{L}$ as seen in figure 20(c), thus clearly demonstrating the effect of the energy transient (which we discussed in $\S 6.4$ ) by having removed this transient from these Lagrangian integrations. For $t>T_{L}, \overline{\left(\boldsymbol{x}(t)-\boldsymbol{x}_{0}\right)^{2}} \sim t$ in agreement with Taylor (1921) (see figure 20c).

The Lagrangian integrations in the quasi-stationary flow without energy transient confirm the existence of the intermediate range of times starting at about $T_{L}$ and lasting for about one decade or so after $T_{L}$. In this range, $\overline{\Delta^{2}} \sim t^{2.45}$ (see figure $20 a, b$ ). The exponent $\gamma$ is close to 2.3 but larger, in fact $\gamma=2.45$ now that the small contamination by the energy transient of the inner end of the time range has been removed. It is worth pointing out that our quasi-steady multi-scale laminar flow stirs and disperses pairs of fluid elements more effectively than a quasi-steady laminar shear flow for which $\gamma=2$. 

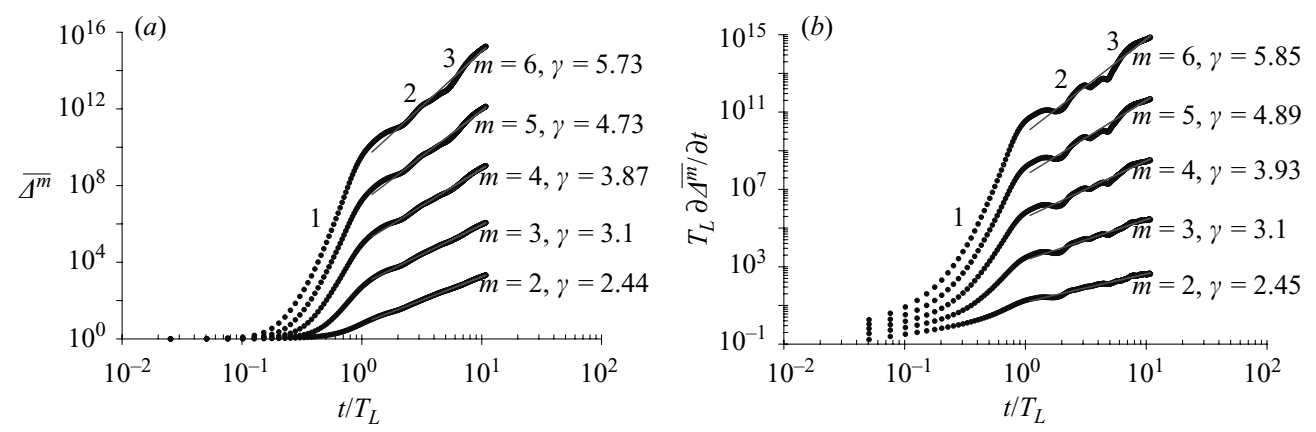

Figure 21. (a) $\overline{\Delta^{m}}$ and its $(b)$ time derivative versus time for various values of $m(2,3,4,5$, 6). The straight lines correspond to power-law fittings with $\overline{\Delta^{m}} \sim t^{\gamma}$, the values of $\gamma$ are given on the graph. (1 619484 pairs.)
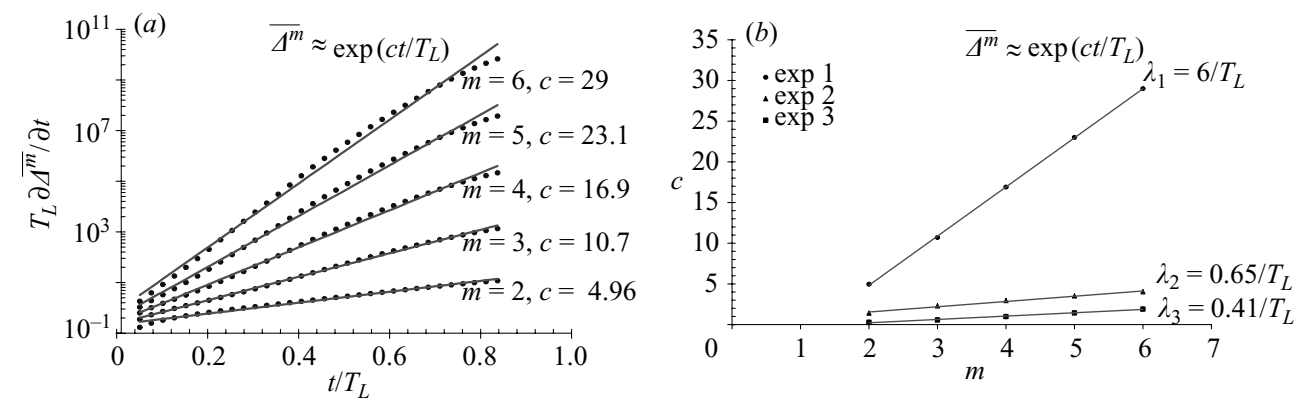

Figure 22. (a) Time derivative of $\overline{\Delta^{m}}$ for various values of $m(2,3,4,5,6)$ and $t<T_{L}$. The straight lines correspond to exponential fittings: $\overline{\Delta^{m}} \sim \exp \left(c t / T_{L}\right)$, the values of $\mathrm{c}$ are given on the graph; $(b)$ evolution of $c$ with $m$ for the three exponential growths indicate by 1,2 and 3 on figure $21 ; c=m \lambda_{i} T_{L}$. (1 619484 pairs.)

\subsubsection{Multi-scale structure of stagnation points and of pair dispersion}

The initial growth (for $t<T_{L}$ ) of pair dispersion is best fitted by an exponential rather than a power law, as shown in figure $22(a)$ where this point is made clear for all moments $\overline{\Delta^{m}}(t)$ of orders $m=2,3,4,5,6$.

In fact, direct inspection of the higher orders of $\overline{\Delta^{m}}(t)$ (see figure 21) reveals the sequential exponential nature of pair separations which is hidden at the lower orders under an apparent power law $\overline{\Delta^{m}}(t) \sim t^{\gamma_{m}}$ (in this notation, $\gamma_{2}=\gamma$ ) in the range of times between $T_{L}$ and about $10 T_{L}$. In figure 21 , we have plotted $\overline{\Delta^{m}}$ and its time derivative. The two plots reveal three bumps increasingly clearly for larger values of $m$. These bumps are indicated by 1,2 and 3 on the plots (figure 21) and, similarly to figure $22(a)$, they can be fitted by local exponential growths $\overline{\Delta^{m}(t)} \sim \exp \left(m \lambda_{i} t\right)$ where $i=1,2,3$ identifies the sequence of exponentials. The fits being obtained for all values of $i$ and all our values of $m$, we can extract $\lambda_{i}$ with some confidence simply by verifying that the exponent $c=m \lambda_{i} T_{L}$ is indeed a linear function of $m$ (see figure $22 b$ ) for the three exponential growths (noted exp1, exp2, exp3 on the figure). We thus obtain the three values of $\lambda_{i}: \lambda_{1} \simeq 6 / T_{L}$ valid for times $t<T_{L} ; \lambda_{2} \simeq 0.65 / T_{L}$ valid for times $T_{L}<t<3 T_{L} ; \lambda_{3} \simeq 0.41 / T_{L}$ valid for times $3 T_{L}<t<8 T_{L}$. These three characteristic scales of stirring seem to correspond to the three scales of forcing, $\lambda_{1}$ corresponding to the smallest scale which has turnover time about equal to $T_{L}, \lambda_{2}$ to the intermediate scale with turnover time about equal to $3 T_{L}$ and $\lambda_{3}$ to the largest 
scale which has a turnover time of the order of $8 T_{L}$ to $10 T_{L}$. It can be checked in figure 21 than these observed turnover times match the inner and outer bounds of the time-ranges of the three sequential exponential growths.

The appearance of three sequential exponential growths in the statistics of pair dispersion can be, in part, accounted for by the relative strengths of these exponentials. The dispersion due to the smallest scales with highest value $\lambda_{1}$ in the $\lambda_{i}$ set takes the lead until a time when the turnover time of these smallest scales is reached beyond which their exponential dispersive action saturates because the flow is quasitwo-dimensional and quasi-stationary. The second exponential growth follows as it becomes dominant only after the first exponential growth has saturated and it lasts until the turnover time of the intermediate scale has been reached. Finally, the third exponential growth emerges as a result of the saturation of the second and lasts until the turnover time of the largest eddies has been reached. These three exponential growths must be linked to the hyperbolic stagnation points generated by the three length scales of EM forcing, and $\lambda_{i}$ for $i=1,2,3$ are presumably the three characteristic strain rates associated with the hyperbolic stagnation points at each one of these scales.

These qualitative observations may be quantified into a simple model of pair dispersion which we now describe. The hyperbolic stagnation points linked to the smallest scales of forcing and motion cause an exponential separation of those pairs which are within the hyperbolic points' area of influence. Hence, $\overline{\Delta^{m}(t)} \sim \Delta_{0}^{m} p_{1} \exp \left(m \lambda_{1} t\right)$ where $\Delta_{0}$ is the initial pair separation and $p_{1}$ is the portion of flow area directly influenced by the smallest-scale hyperbolic points. This exponential growth holds for $0<t<t_{1}$ where $t_{1} \sim \lambda_{1}^{-1}$ and $t_{1} \sim l_{2} / u_{r m s}$, assuming that $u_{r m s}$ is the characteristic flow velocity at the smallest scale $l_{2}$ (M10). In the subsequent time range $t_{1}<t<t_{2}$, the pair separation action of the smallest-scale hyperbolic stagnation points saturates and the pair separation process is taken over by the intermediate-scale stagnation points. Hence, $\overline{\Delta^{m}(t)} \sim \overline{\Delta^{m}\left(t_{1}\right)} p_{2} \exp \left(m \lambda_{2} t\right)$ for $t_{1}<t<t_{2}$ where $p_{2}$ is the portion of flow area directly influenced by the hyperbolic points linked to the length-scale $l_{1}$ (M40), $t_{2} \sim \lambda_{2}^{-1}$ and $t_{2} \sim l_{1} / u_{r m s}$, assuming again that $u_{r m s}$ is the characteristic flow velocity at the smallest scale $l_{1}$. Indeed, because of our particular fractal forcing, the characteristic velocities at different scales of forcing are found not to be too different, but there are some variations which, however, do not appear to be systematic.

In the final time range, $t_{2}<t<t_{3}$, the pair separation action of the intermediate scale hyperbolic points saturates and it is the largest-scale stagnation point that takes over the final separation process. Hence, $\overline{\Delta^{m}(t)} \sim \overline{\Delta^{m}\left(t_{2}\right)} p_{3} \exp \left(m \lambda_{3} t\right)$ for $t_{2}<t<t_{3}$ where $p_{3}$ is the portion of flow area directly influenced by the hyperbolic point linked to the length-scale $l_{0}(\mathrm{M} 160), t_{3} \sim \lambda_{3}^{-1}$ and $t_{3} \sim l_{0} / u_{r m s}$. Note that $p_{i} \sim\left(l_{2-i}\right)^{2-D_{s}}$ for $i=1,2,3$, where $D_{s}$ is the fractal dimension of the stagnation points (see $\S 5.1$ ), and that $l_{i} \sim R^{-i} l_{0}$ for $i=1,2,3$.

The upshot is that, for $i=1,2,3, \overline{\Delta^{m}\left(t_{i}\right)} \sim\left(R^{(i-3)^{2}+(i-3)}\right)^{1-D_{s} / 2} \exp (B m i)$ where $B$ is defined by $t_{i}=B \lambda_{i}^{-1}$ and $t_{i}=A R^{i-3} l_{0} / u_{\text {rms }}$ ( $A$ and $B$ are dimensionless constants). It follows that

$$
\overline{\Delta^{m}\left(t_{i}\right)} \sim\left(u_{r m s} t_{i} / l_{0}\right)^{m \frac{B}{\ln (R)}}+C\left(1-D_{s} / 2\right)
$$

where we have replaced $1+\left(\left(\ln \left(u_{r m s} t_{i} / l_{0}\right)-A\right) / \ln (R)\right)^{2}$ by an approximate constant $C$. Indeed, over the range $t_{1}$ to $t_{3}$, this 'constant' does not vary much if $A$ is small enough. It can therefore be expected that

$$
\overline{\Delta^{m}(t)} \sim t^{\gamma_{m}}
$$

with $\gamma_{m}=m(B / \ln (R))+C\left(1-D_{s} / 2\right)$ is a good approximation in the range $t_{1}<t<t_{3}$, particularly for small enough values of $m$. Larger values of $m$ amplify the oscillations 
around $t^{\gamma_{m}}$ which are caused by the sequence of exponentials underpinning the dispersion process. Power-law fits of $\overline{\Delta^{m}(t)}$ in this range are given in figure $21(a)$, but they are very sensitive to the way the fit is taken, particularly for $m=4,5,6$. A type of fit slightly different from the one in figure 21 designed to remove the significant effect of the bump at $T_{L}$ (i.e. we fit from $2 T_{L}$ until $10 T_{L}$ ) leads to $\gamma_{2} \approx 2.5$ and $\gamma_{3} \approx 3.1$ as in figure $21(a)$, but $\gamma_{4} \approx 3.4, \gamma_{5} \approx 3.9$ and $\gamma_{6} \approx 4.3$ unlike the fits in figure 21 $(a)$. These values are approximated well by $\gamma_{m}=1.5+m / 2$ (with increasing error bars for increasing $m$, as the disagreement with the fits of figure 21(a) attests to in the cases $m=4,5,6)$, and our formula $\gamma_{m}=m(B / \ln (R))+C\left(1-D_{s} / 2\right)$ can match this estimation by taking $B=(\ln R) / 2=\ln 2$ and $C=2$. This value of $C$ is realized for $A \approx \ln R-1=\ln 4-1$, in which case $C$ is indeed an approximate constant in the range $t_{1}<t<t_{3}$. Hence,

$$
\gamma_{m}=2-D_{s}+m / 2 \text {. }
$$

In conclusion, the multi-scale spatial distribution of stagnation points, which seems to be responsible for the power-law shape of the energy spectrum, is also responsible for the pair dispersion properties of this quasi-stationary multi-scale laminar flow. Hyperbolic stagnation points at different length scales cause pairs to separate exponentially at rates that are a decreasing function of length scale. This results in a sequence of exponential pair separation processes which, for low orders $m$, aggregate into approximate power laws $\overline{\Delta^{m}(t)} \sim t^{\gamma_{m}}$ for low enough orders $m$ in the intermediate range of times determined by the hyperbolic points' strain rates. The exponents $\gamma_{m}$ seem to have a linear dependence on $m$ with coefficients which depend on the multi-scale nature of the forcing, i.e. which depend on $R$ and $D_{s}$.

\section{Conclusion}

We have been able to generate and control over a wide range of scales a class of multi-scale laminar flows with continuous power-law spectra and Lagrangian statistics in agreement with Taylor's (1921) ballistic and Brownian regimes. Furthermore, the Lagrangian pair statistics of these flows have some Richardson-like properties in the sense that the mean square pair separation grows like an approximate power law $t^{\gamma}$ with a 'Richardson exponent' $\gamma$ that is clearly larger than 2 . The other sense in which these pair statistics are Richardson-like is that they seem to respond to a localityin-scale principle, as Richardson (1926) indeed hypothesized for fully developed turbulent flows. The pairs respond locally to different length scales according to the stage in their average growth, starting from the smallest scales and developing all the way through to the largest. Indeed, it has been possible to demonstrate that the apparent power law $t^{\gamma}$ belies a sequence of local exponential growths each related to stagnation points linked to a particular length scale. Lagrangian pairs experience a first exponential growth as a result of hyperbolic stagnation-point action at the smallest scales, then another exponential growth as a result of hyperbolic stagnationpoint action at the subsequent length scale, and so on until the hyperbolic stagnation points of the largest scales have been reached. This is a locality-in-scale process directly linked to the multi-scale topology of the flow.

These multi-scale laminar flows can be generated and fully controlled over a wide range of Reynolds numbers and with separate control over Reynolds number and range of scales where $E(k)$ is a power law. We are using a fractal-like (and therefore multi-scale) electromagnetic Lorentz body forcing of a quasi-two-dimensional layer of brine in a rig of record dimensions for its kind so as to shield the forced flow from 
boundary effects. This forcing is controlled in scales (space and time) and intensities and is currently being further developed in ongoing work by us in our laboratory to allow for well-designed time-dependencies of the forcing, various changes in the fractal properties of the spatial distribution, sizes and even potential movements of the magnets. In particular, various breaks of symmetry in the magnets' geometry are envisaged which might also break the relation $p+D_{s}=3$ and modify the relation between $\gamma$ and $D_{s}$ too (for the quasi-stationary flow driven by a current $I=0.1 \mathrm{~A}$, we have argued that $\gamma=3-D_{s}$, see equation (6.1)). The results presented in this paper support $p+D_{s}=3$ and $\gamma=3-D_{s}$, but they do so only for one magnet set-up. It is not easy to modify the magnet set-up at will without inordinate expenditure of time and effort, but we are currently modifying our rig to make this possible, and then it will also be possible to test more fully the validity and limitations of $p+D_{s}=3$ and $\gamma=3-D_{s}$ for various values of $D_{s}$ and geometries. We do expect, for example, $\gamma$ to be larger than $3-D_{s}$ and perhaps closer to the prediction (1.3) when the forcing, and therefore also the multi-scale flow, will be made time-dependent. Nevertheless, the results in this paper do go some way in demonstrating in the laboratory how a power-law energy spectrum and Richardson-like pair statistics can be generated from many elementary local-in-scale eddy features (such as stagnation points) and their corresponding local-in-scale processes (exponential pair separations).

We control the multi-scale topology of the flow by magnet pairs which produce forced stagnation points at chosen locations on a fractal-like set. The multi-scale topology of our flows is found not to change with Reynolds number, i.e. it remains the same for a broad range of flow intensities. However, the detailed streamline structure, by which we mean streamline shapes and sizes of closed streamlines, is sensitive to the balance between bottom friction and intensity of forced advection. Related to this, when the Reynolds number is not large enough, oscillations are evident around the power-law energy spectrum which are, of course, due to the log-periodic nature of the forcing. As the Reynolds number increases, interferences between scales increase as bottom-friction effects weaken and these oscillations dim out too.

To our knowledge it is the first time that multi-scale electromagnetic control of the energy spectrum and of Lagrangian dispersion via fractal-like forcing is realized in the laboratory. In fact, even if laminar, the multi-scale flows that we have created are turbulent-like in various ways described in this paper, and should be more so with time-dependent forcing.

We hope that this work will pave the way for new research in an entirely new class of flows with potentially important consequences for mixing and multi-scale flow control as well as for the understanding of how topology, dynamics and (Eulerian and Lagrangian) statistics relate in general and in turbulent flows in particular.

We are grateful for financial support from The Leverhulme Trust and the Royal Society. We also thank Dimitrios Kolokotronis for his help with the PIV software.

\section{Appendix. Fractal dimension of the forcing}

The fractal-like structure of stagnation points according to flow scales is given in equation (A 1) (cf. Davila \& Vassilicos 2003; Goto \& Vassilicos 2004):

$$
N_{s}=C_{s}\left(\frac{L_{0}}{L}\right)^{d}(L / \eta)^{D_{s}}+C_{b},
$$


$N_{s}$ is the number of stagnation points; $d$ is the spatial dimension; $D_{s}$ is the fractal dimension of the stagnation points distribution; $L_{0}$ is the size of the bulk/flow; $L$ is the integral length scale; $\eta$ is a length threshold (greater than Kolmogorov scale); $C_{s}$ is a bulk parameter which represent the influence of the outer scales to the small scales. $C_{b}$ takes into account the influence of the large-scale structure and boundaries conditions. $C_{b}$ is not transmitted via the self-similar process as is $C_{s}$ and thus represent the outer scales information which is lost at the small scales. When $L \gg \eta$ (i.e. large Reynolds number), this correction is not important. It is only important to have an exact equation when looking at the distribution of the stagnation points for $\eta \sim L$. As we target here the generation and control of a multi-scale flow, i.e. from the large scale $(\gtrsim L)$ to the small scales, we introduce and use (see §5.2) this minor correction.

We force the flow in an 8 in 8 shape where scales are successively inserted inside each other. Figure 1 illustrates this construction. The forcing is based on controlling the saddle points at the heart of the 8. In a similar way to (A 1) we write the number of 'forced saddle points' (or scale of forcing), where $n$ is the iteration number and $r=\eta_{n} / \eta_{n+1}$, as:

$$
N_{s_{f}}=C_{s_{f}} r^{n D_{s_{f}}}+C_{b_{f}} .
$$

The number of forcing scales based on the figure 1 scheme is $2^{n+1}-1$. This leads to $C_{b_{f}}=-1$ and so $C_{s_{f}}=2$, as $C_{s_{f}}+C_{b_{f}}=1$ for $n=0$. The fractal dimension of the forcing, $D_{s_{f}}$ is thus dependent on the choice of the successive scales of forcing:

$$
D_{s_{f}}=\ln (2) / \ln (r) \text {. }
$$

The forcing follows figure $2(b)$. The corresponding distribution of forced stagnation points is $N_{s}=2^{n+2}-3$. this leads to $C_{b_{f}}=-3$ and $C_{s_{f}}=4$. The fractal dimension is also given by equation (A 3). We chose $r=4$ for the present experiments, so the fractal dimension of the forcing is $D_{s_{f}}=0.5$. It should be noticed than without the large-scale correction of equation (A 2) the fractal dimension of the forcing computed with $C_{b_{f}}=0$ between iteration $i$ and $j$ is given by:

$$
D_{s_{f}}=\frac{\ln \left(N_{s_{f}} / N_{s_{f}}\right)}{\ln \left(\eta_{j} / \eta_{i}\right)}
$$

This leads to a fractal dimension dependent of the number of iteration (and thus the chosen $i$ and $j$ ). Nevertheless, if $j>i \gg 1$, this relation also leads to $D_{s_{f}}=0.5$. As in the present experiments, the number of iterations is limited to 3 (which is already challenging), this definition of the fractal dimension would gives $D_{s_{f}}=0.611$ for the 8 in 8 schemes and $D_{s_{f}}=0.689$ if not taking into account that the forcing constitutes a double 8 structure.

\section{REFERENCES}

Akoun, G. \& Yonnet, J.-P. 1984 3D analytical calculation of the forces exerted between two cuboidal magnets. IEEE Trans. Magnet. 20, 1962-1964.

Biferale, L., Lanotte, A. S. \& Toschi, F. 2004 Effects of forcing in three-dimensional turbulent flows. Phys. Rev. Lett. 92, 094503.

Boffeta, G., Cenedese, A., Espa, S. \& Musacchio, S. 2005 Experimental study of two-dimensional enstrophy cascade. Europhys. Lett. arXiv : nlin.CD $\backslash 0505034 \mathrm{~V} 1$.

Boffeta, G. \& Sokolov, I. M. 2002 Relative dispersion in fully developed turbulence: the Richardson's law and intermittency corrections. Phys. Rev. Lett. 88, 094501.

Cardoso, O., Marteau, D. \& Tabeling, P. 1994 Quantitative experimental study of the free decay of quasi-two-dimensional turbulence. Phys. Rev. E 49, 454-461. 
Clercx, H. J. H. \& van Heisst, G. J. F. 2000 Energy spectra for decaying 2D turbulence in a bounded domain. Phys. Rev. Lett. 85, 0031-9007.

Clercx, H. J. H., van Heisst, G. J. F. \& Zoeteweis, M. L. 2003 Quasi-two-dimensional turbulence in shallow fluid layers: the role of bottom friction and fluid layer depth. Phys. Rev. E 67, 066303.

Cuypers, Y., Maurel, A. \& Petitjeans, P. 2003 Vortex burst as a source of turbulence. Phys. Rev. Lett. 91, 194502-1.

Davidson, P. A. 2001 An Introduction to Magnetohydrodynamics. Cambridge University Press.

Davidson, P. A. 2004 Turbulence: an Introduction for Scientists and Engineers. Oxford University Press.

Davila, J. \& Vassilicos, J. C. 2003 Richardson pair diffusion and the stagnation point structure of turbulence. Phys. Rev. Lett. 91, 144501.

Dolzhanskit, F. V., Krymov, V. A. \& Manin, D. Yu. 1992 An advanced experimental investigation of quasi-two-dimensional shear flows. J. Fluid Mech. 241, 705-723.

Falkovich, G., Gawedzki, K. \& Vergassola, M. 2001 Particles and fields in fluid turbulence. Rev. Mod. Phys. 73, 913-975.

Frisch, U. 1995 Turbulence. Cambridge University Press.

Fung, J. C. H., Hunt, J. C. R., Malik, N. A. \& Perkins, R. J. 1992 Kinematic simulation of homogeneous turbulence by unsteady random Fourrier modes. J. Fluid Mech. 236, 281-318.

Fung, J. C. H. \& Vassilicos, J. C. 1998 Two-particle dispersion in turbulentlike flows. Phys. Rev. E 57, 1677-1690.

Goto, S., Osborne, D. R., Vassilicos, J. C. \& Haigh, J. D. 2005 Acceleration statistic as measures of statistical persistence of streamlines in isotropic turbulence. Phys. Rev. E 71, 015301(R).

Goto, S. \& Vassilicos, J. C. 2004 Particle pair diffusion and persistent streamline topology in two-dimensional turbulence. New J. Phys. 6, 1-35.

Hurst, D. \& VAssiLicos, J. C. 2006 Scalings and decay of fractal generated turbulence. Phys. Fluids (submitted).

IsHIHARA, T. \& KANEDA, Y. 2002 Relative diffusion of a pair of fluid particles in the inertial subrange of turbulence. Phys. Fluids 14, L69.

Julien, M. C., Paret, J. \& Tabeling, P. 1999 Richardson pair dispersion in two-dimensional turbulence. Phys. Rev. Lett. 82, 2872-2875.

Khan, M. A. I. \& VAssiLicos, J. C. 2001 Scalings of scalar structure in a velocity field with coherent vortical structures. Phys. Rev. E 65, 016304.

Kolokotronis, D. 2006 Experimental investigation of the internal flow field of model fuel injectors. $\mathrm{PhD}$ thesis. Mech. Engng Dept, Imperial College London.

Kraichnan, R. H. 1967 Inertial range transfer in two-dimensional turbulence. Phys. Fluids 10, $1417-1423$.

Lundgren, T. S. 1982 Strained spiral vortex model for turbulent fine structure. Phys. Fluids 25, 2193-2203.

Mathieu, J. \& Scott, J. 2000 An Introduction to Turbulent Flows. Cambridge University Press.

Mazzi, B. \& VassiLicos, J. C. 2004 Fractal generated turbulence. J. Fluid Mech. 502, 65-87.

Moffatt, H. K. 2001 The topology of scalar fields in 2D and 3D turbulence. IUTAM Symp. on Geometry and Statistics of Turbulence, Kluwer, pp. 13-22.

Moreau, R. 1991 Magnetohydrodynamics. Kluwer.

Nicolleau, F. \& YU, G. 2004 Two-particle diffusion and locality assumption. Phys. Fluids. 16, 2309-2321.

Oвuкноv, A. M. 1941 Spectral energy distribution in turbulent flow. Izv. Akad. Nauk SSSR 5, 453-566.

OreY, S. 1970 Gaussian sample functions and the Hausdorff dimension of level crossings. Z. Wahrscheinlichkeitstheorie 15, 249.

Osborne, D. R., Vassilicos, J. C., Sung, K. \& Haigh, J. D. 2006 Fundamentals of pair diffusion in kinematic simulations of turbulence. Phys. Rev. E (to appear).

OTt, S. \& MANN, J. 2000 An experimental investigation of the relative diffusion of particles pairs in three-dimensional turbulent flow. J. Fluid Mech. 422, 207-223.

Ottino, J. M. 1989 The Kinematics of Mixing: Stretching, Chaos, and Transport. Cambridge University Press. 
Paret, J., Marteau, D., Paireau, O. \& Tabeling, P. 1997 Are flows electromagnetically forced in thin stratifed layers two-dimensional? Phys. Fluids 9, 3102-3104.

Paret, J. \& Tabeling, P. 1997 Experimental observation of the two-dimensional inverse energy cascade. Phys. Rev. Lett. 79, 4162-4165.

Perry, A. E. \& Chong, M. S. 1987 A description of eddying motions and flow patterns using critical-point concepts. Annu. Rev. Fluid Mech. 19, 125-155.

Pope, S. B. 2000 Turbulent Flows. Cambridge University Press.

Queiros-Conde, D. \& VAssilicos, J. C. 2001 Turbulent wakes of 3D fractal grids. In Intermittency in Turbulent Flows. Cambridge University Press.

Raffel, M., Willert, C. \& Kompenhans, J. 1998 Particle Image Velocimetry, A Practical Guide. Springer ISBN 3-540-63683-8.

Richardson, L. F. 1926 Atmospheric diffusion shown on a distance-neighbour graph. Proc. R. Soc. Lond. A 110, 709-737.

Rossi, L. 2001 Contrôle électromagnétique d'écoulement en eau de mer. PhD thesis, Université Joseph Fourier, Grenoble.

Rossi, L., Hascoët, E., Vassilicos, J. C. \& Hardalupas, Y. 2005 2D fractal flow generated by electromagnetic forcing: laboratory experiments and numerical simulations. Turb. Shear Flow Phenom. 4, 485-490.

Rothstein, D., Henry, E. \& Gollub, J. P. 1999 Persistent patterns in transient chaotic fluid mixing. Nature 401, 770-772.

Satijn, M. P., Cense, A. W., Verzicco, R., Clercx, H. J. H. \& Van Heijst, G. J. F. 2001 Three dimensionnal structure and decay properties of vortices in shallow fluid layers. Phys. Fluids 13, 2343-2353.

Sommeria, J. 1986 Experimental study of the two-dimensional inverse energy cascade in a square box. J. Fluid Mech. 170, 1932-1945.

Staicu, A., Mazzi, B., Vassilicos, J. C. \& Van DE Water, W. 2003 Turbulent wakes of fractal objects. Phys. Rev. E 67, 066306

Tabeling, P. 2002 Two-dimensional turbulence: a physicist approach. Phys. Rep. 362, 1-62.

TAYlor, G. I. 1921 Diffusion by continuous movement. Proc. Lond. Math. Soc. 20, 196.

Thibault, J. P. \& Rossi, L. 2003 Electromagnetic flow control: characteristic numbers and flow regimes of a wall-normal actuator. J. Phys. D: Appl. Phys. 36, 2559-2568.

VAssilicos, J. C. 2002 Mixing in vortical, chaotic and turbulent flows. Phil. Trans. R. Soc. Lond. A 360, 2819-2837.

Voth, G. A., Haller, G. \& Gollub, J. P. 2002 Experimental measurements of stretching fields in fluid mixing. Phys. Rev. Lett. 88, 254501.

Williams, B. S., Marteau, D. \& Gollub, J. P. 1997 Mixing of a passive scalar in magnetically forced two-dimensional turbulence. Phys. Fluids 9, 2061-2080. 